\title{
Robust Multimode Function Synchronization of Memristive Neural Networks with Parameter Perturbations and Time-Varying Delays
}

\author{
Wei Yao, Chunhua Wang, Yichuang Sun, Senior Member, IEEE, and Chao Zhou
}

\begin{abstract}
Currently, some works on studying complete synchronization of dynamical systems are usually restricted to its two special cases: power-rate synchronization and exponential synchronization. Therefore, how to give a generalization of these types of complete synchronization by mathematical expression is an open question which needs to be urgently solved. To begin with, this paper proposes multimode function synchronization by mathematical expression for the first time, which is a generalization of exponential synchronization, power-rate synchronization, and logarithmical synchronization and so on. Moreover, two adaptive controllers are designed to achieve robust multimode function synchronization of memristive neural networks (MNNs) with mismatched parameters and uncertain parameters. Each adaptive controller includes function $r(t)$ and update gain $\sigma$. By choosing different types of $r(t)$, multiple types of complete synchronization including power-rate synchronization and exponential synchronization can be obtained. And update gain $\sigma$ can be used to adjust the speed of synchronization. Therefore, our results enlarge and strengthen the existing results. Two examples are put forward to verify the validity of our results.
\end{abstract}

Index Terms-Memristive neural networks (MNNs), complete synchronization, multimode function synchronization, adaptive controller, mismatched parameters, uncertain parameters.

\section{INTRODUCTION}

$\mathbf{I}$ N 1971, memristor was first assumed by Chua [1], and the nanoscale material object of memristor was achieved by HP laboratory in 2008 [2]. Due to the excellent properties, such as nonvolatile memory and low power, memristor has very broad application prospects in chaotic circuits, neural networks and so on [3-12].

Memristive neural network (MNN) can be designed by replacing resistor of neural network with memristor to imitate the synapse [12-14], [18, 19], [24-41]. MNN has broad applications in some fields [12-14], such as higher brain functions, logical operations, image processing, etc. Hence, it is necessary to research the dynamical properties of MNN [16-41], one of which is synchronization. Recently, there are many works on synchronization problems of dynamical

Manuscript received April 4, 2019; revised September 10, 2019; revised December 4, 2019. This work was supported in part by the National Natural Science Foundation of China under Grant 61971185, in part by the Science and Technology Planning Project of Hunan Province, China under Grant 2017GK4009, and in part by the Open Fund Project of Key Laboratory in Hunan Universities under Grant 18K010. (Corresponding author: Chunhua Wang.)

Wei Yao, Chunhua Wang and Chao Zhou are with the College of Information Science and Engineering, Hunan University, Changsha, 410082, China (e-mail:wch1227164@hnu.edu.cn).

Yichuang Sun is with the School of Engineering and Computer Science, University of Hertfordshire, Hatfield AL10 9AB, U.K. systems [16]-[46], because of its broad application prospects $[15,16]$, including information processing and secure communication. Up to now, some types of concepts of synchronization were investigated, for example, power-rate synchronization [42, 43], exponential synchronization [26-31], [35], [37], anti-synchronization [33, 34], projective synchronization [36], function projective synchronization [24], lag synchronization [17-19], cluster synchronization [20], multisynchronization [21-23] and so on. Currently, synchronization of dynamical systems can be realized by using feedback control [26-37]. For instance, anti-synchronization with linear feedback control was investigated in [33] and [34]. Power-rate synchronization by impulsive control was addressed in [42]. Exponential synchronization was studied via state feedback control [26, 27], [35], pinning control [28], delay-dependent feedback control [30], state feedback control or adaptive feedback control [31], [37]. In [36], finite-time projective synchronization by linear feedback control was achieved. Different feedback controllers are designed to achieve different types of synchronization and meet different needs. For instance, if a general type of synchronization is required, projective synchronization [36] and function projective synchronization [24] are suited. Projective synchronization (or function projective synchronization) can be regarded as a general form of complete synchronization and anti-synchronization. Actually, power-rate synchronization [42, 43] and exponential synchronization [26-31], [35], [37] are two special cases of complete synchronization. Currently, complete synchronization has been extensively investigated in some literatures [26-31], [35], [37], [42, 43]. However, these works are usually restricted to power-rate synchronization and exponential synchronization. Therefore, how to generalize these types of complete synchronization by mathematical expression is an open question which needs to be urgently solved.

Because of environmental disturbances and dependence on state for parameters of MNNs, we usually cannot acquire parameter values without deviation and the parameters of drive and response MNNs may be mismatched [38-41]. Uncertain parameters and mismatched parameters of MNNs may lead to instability and some unpredictable influence for the systems. Therefore, the effect of uncertain parameters and mismatched parameters cannot be neglected. In recent years, there are some researches on robust synchronization of MNNs with uncertain parameters or mismatched parameters [38-41]. For example, Li et al. investigated robust synchronization of MNNs with mismatched coefficients and mismatched time- 
varying delays [38]. Finite-time robust synchronization and asymptotical robust synchronization of MNNs were studied with mismatched parameters in [40] and [39], respectively. When there exist uncertain parameters in multiple MNNs, the robust synchronization problem was investigated in [41]. However, there is no work on robust synchronization of drive and response MNNs with mismatched parameters and uncertain parameters.

Inspired by the above-mentioned discussions, we focus attention on robust complete synchronization of drive and response MNNs with mismatched parameters and uncertain parameters. For the purpose of investigating complete synchronization of drive/response dynamical systems, the multimode function synchronization is proposed, which can generalize some existing definitions of complete synchronization including power-rate synchronization and exponential synchronization. This paper will deal with the robust multimode function synchronization problem between drive MNN system and response MNN system. Robust multimode function synchronization means that the states of drive and response MNN systems with parameter perturbations are identical via multiple convergence methods. It is worth mentioning that powerrate synchronization $[42,43]$ and exponential synchronization [26-31], [35], [37] can be viewed as two special cases of multimode function synchronization. Compared with powerrate synchronization $[42,43]$ and exponential synchronization [26-31], [35], [37], multimode function synchronization is more convenient to be used in practical applications and can meet more requirements due to its flexible and multiple types of complete synchronization. Therefore, multimode function synchronization enlarges the existing results and has generality. Moreover, this paper designs two adaptive controller$\mathrm{s}$ to achieve robust multimode function synchronization of MNNs with parameter perturbations and time-varying delays. Compared with linear feedback control [33], [34], [36] and state feedback control [26, 27], [31], [35], [37], [39], adaptive control is more flexible. The main contributions can be summarized as follows. 1) Multimode function synchronization, which is a generalization of power-rate synchronization, exponential synchronization, and logarithmical synchronization and so on, is proposed for the first time. Thus multimode function synchronization is suitable for power-rate synchronization and exponential synchronization. 2) Two adaptive controllers are designed to achieve robust multimode function synchronization of MNNs with mismatched parameters and uncertain parameters. 3) Each adaptive controller in this paper includes function $r(t)$ and update gain $\sigma$. Function $r(t)$ can be freely chosen, such as exponential function, polynomial function, logarithmical function, then corresponding robust complete synchronization can be obtained. And update gain $\sigma$ can be applied to adjust synchronization speed according to the practical needs.

The rest of the paper is organized as follows. In Section II, MNNs with parameter perturbations and the definition of multimode function synchronization are introduced. Two adaptive controllers are designed to realize robust multimode function synchronization of MNNs in Section III. Section IV presents numerical simulations to verify the effectiveness of the obtained results. Finally, conclusions are given in Section V.

\section{Memristive Neural Network System And PRELIMINARIES}

Notations: For vector $\vartheta=\left(\vartheta_{1}, \vartheta_{2}, \ldots, \vartheta_{l}\right)^{T} \in \Re^{l}$, $\|\vartheta\|=\left[\sum_{m=1}^{l} \vartheta_{m}^{2}\right]^{\frac{1}{2}} \cdot \Gamma_{1}$ and $\Gamma_{2}$ denote $\left|x_{m}(t)\right| \leq$ $\kappa_{m}$ and $\left|x_{m}(t)\right|>\kappa_{m}$, respectively; $\Omega_{1}$ and $\Omega_{2}$ denote $\left|y_{m}(t)\right| \leq \kappa_{m}$ and $\left|y_{m}(t)\right|>\kappa_{m}$, respectively; and $\kappa_{m}$ represents switching threshold. $\hat{\varsigma}_{m z}=\max \left\{\left|\dot{\varsigma}_{m z}\right|,\left|\ddot{\zeta}_{m z}\right|\right\}$; $\hat{\rho}_{m z}=\max \left\{\left|\dot{\rho}_{m z}\right|,\left|\ddot{\rho}_{m z}\right|\right\} ; \hat{\beta}_{m z}=\max \left\{\left|\dot{\beta}_{m z}\right|,\left|\ddot{\beta}_{m z}\right|\right\}$ and $\hat{\omega}_{m z}=\max \left\{\left|\dot{\omega}_{m z}\right|,\left|\ddot{\omega}_{m z}\right|\right\} ;$ where $\dot{\varsigma}_{m z}, \ddot{\varsigma}_{m z}, \dot{\rho}_{m z}, \ddot{\rho}_{m z}, \dot{\beta}_{m z}$, $\ddot{\beta}_{m z}, \dot{\omega}_{m z}$ and $\ddot{\omega}_{m z}$ are constants; $m, z=1,2, \ldots, l$.

We consider MNN system with time-varying delays as:

$$
\begin{gathered}
\dot{x}_{m}(t)=-c_{m} x_{m}(t)+\sum_{z=1}^{l} \varsigma_{m z}\left(x_{m}(t)\right) f_{z}\left(x_{z}(t)\right) \\
\quad+\sum_{z=1}^{l} \rho_{m z}\left(x_{m}(t)\right) g_{z}\left(x_{z}\left(t-\tau_{z}(t)\right)\right)+I_{m}(t), \\
t \geq 0, \quad m=1,2, \ldots, l,
\end{gathered}
$$

where the self-inhibition $c_{m}$ of neuron $x_{m}(t)$ is positive constant; $f_{z}($.$) and g_{z}($.$) are activation functions; \varsigma_{m z}\left(x_{m}(t)\right)$ and $\rho_{m z}\left(x_{m}(t)\right)$ are memristive connection weights; $\tau_{z}(t)$ is the time-varying delay and $0 \leq \tau_{z}(t) \leq \tau$ ( $\tau$ is a positive constant); and $I_{m}(t)$ is the external input.

According to the simple memeristor model [36], the memristive connection weights $\varsigma_{m z}\left(x_{m}(t)\right)$ and $\rho_{m z}\left(x_{m}(t)\right)$ can be written as:

$$
\varsigma_{m z}\left(x_{m}(t)\right)= \begin{cases}\dot{\varsigma}_{m z}, & \Gamma_{1} \\ \ddot{\varsigma}_{m z}, & \Gamma_{2}\end{cases}
$$

and

$$
\rho_{m z}\left(x_{m}(t)\right)= \begin{cases}\dot{\rho}_{m z}, & \Gamma_{1} ; \\ \ddot{\rho}_{m z}, & \Gamma_{2}\end{cases}
$$

As described above, due to environmental disturbances and dependence on state for parameters of MNNs, there inevitably exist mismatched parameters and uncertain parameters in MNNs in reality. Thus, the MNN system with time-varying delays in (1) can be rewritten as a more realistic one.

$$
\begin{aligned}
& \dot{x}_{m}(t)=-c_{m} x_{m}(t)+\sum_{z=1}^{l}\left[\varsigma_{m z}\left(x_{m}(t)\right)+\Delta \varsigma_{m z}(t)\right] f_{z}\left(x_{z}(t)\right) \\
& \quad+\sum_{z=1}^{l}\left[\rho_{m z}\left(x_{m}(t)\right)+\Delta \rho_{m z}(t)\right] g_{z}\left(x_{z}\left(t-\tau_{z}(t)\right)\right)+I_{m}(t), \\
& t \geq 0, \quad m=1,2, \ldots, l,
\end{aligned}
$$

where $\Delta \varsigma_{m z}(t)$ and $\Delta \rho_{m z}(t)$ represent the uncertain parameters. The initial condition is $x(s)=\phi(s)=$ $\left(\phi_{1}(s), \phi_{2}(s), \ldots, \phi_{l}(s)\right)^{T}$. Similar to paper [41], uncertain parameters $\Delta \varsigma_{m z}(t)$ and $\Delta \rho_{m z}(t)$ are bounded, namely,

$$
\left|\Delta \varsigma_{m z}(t)\right| \leq \mu_{m z},\left|\Delta \rho_{m z}(t)\right| \leq v_{m z},
$$

where $\mu_{m z}$ and $v_{m z}$ are positive constants. 
Set (4) as the drive MNN, then the corresponding realistic response $\mathrm{MNN}$ can be represented as:

$$
\begin{aligned}
& \dot{y}_{m}(t)=-c_{m} y_{m}(t)+\sum_{z=1}^{l}\left[\omega_{m z}\left(y_{m}(t)\right)+\Delta \omega_{m z}(t)\right] f_{z}\left(y_{z}(t)\right) \\
& +\sum_{z=1}^{l}\left[\beta_{m z}\left(y_{m}(t)\right)+\Delta \beta_{m z}(t)\right] g_{z}\left(y_{z}\left(t-\tau_{z}(t)\right)\right) \\
& +I_{m}(t)+u_{m}(t), \quad t \geq 0, \quad m=1,2, \ldots, l,
\end{aligned}
$$

where $u_{m}(t)$ represents appropriate feedback control; $\omega_{m z}\left(y_{m}(t)\right)$ and $\beta_{m z}\left(y_{m}(t)\right)$ denote memristive connection weights; $\Delta \omega_{m z}(t)$ and $\Delta \beta_{m z}(t)$ are the uncertain parameters. The initial condition is $y(s)=\varphi(s)=\left(\varphi_{1}(s), \varphi_{2}(s), \ldots\right.$, $\left.\varphi_{l}(s)\right)^{T}$. Similarly, $\omega_{m z}\left(y_{m}(t)\right)$ and $\beta_{m z}\left(y_{m}(t)\right)$ can be written as:

$$
\begin{aligned}
& \omega_{m z}\left(y_{m}(t)\right)= \begin{cases}\dot{\omega}_{m z}, & \Omega_{1} ; \\
\ddot{\omega}_{m z}, & \Omega_{2} ;\end{cases} \\
& \beta_{m z}\left(y_{m}(t)\right)= \begin{cases}\dot{\beta}_{m z}, & \Omega_{1} ; \\
\ddot{\beta}_{m z}, & \Omega_{2} ;\end{cases}
\end{aligned}
$$

Uncertain parameters $\Delta \omega_{m z}(t)$ and $\Delta \beta_{m z}(t)$ are bounded, namely,

$$
\left|\Delta \omega_{m z}(t)\right| \leq \chi_{m z},\left|\Delta \beta_{m z}(t)\right| \leq \Upsilon_{m z}
$$

where $\chi_{m z}$ and $\Upsilon_{m z}$ are positive constants.

Remark 1: Combining drive MNN (4) and response MNN (5), two types of parameter perturbations can be obtained. The first type is mismatched parameters. In this paper, parameters satisfy the following inequalities: $\varsigma_{m z}\left(x_{m}(t)\right) \neq$ $\omega_{m z}\left(y_{m}(t)\right)$ and $\rho_{m z}\left(x_{m}(t)\right) \neq \beta_{m z}\left(y_{m}(t)\right)$. Hence, parameters $\omega_{m z}\left(y_{m}(t)\right)$ and $\beta_{m z}\left(y_{m}(t)\right)$ of the response MNN (5) are mismatched with $\varsigma_{m z}\left(x_{m}(t)\right)$ and $\rho_{m z}\left(x_{m}(t)\right)$ of the drive MNN (4), respectively. The second type is uncertain parameters. $\Delta \varsigma_{m z}(t), \Delta \rho_{m z}(t)$ in drive MNN (4) and $\Delta \omega_{m z}(t)$, $\Delta \beta_{m z}(t)$ in response MNN (5) are uncertain parameters.

Let the synchronization error $e_{m}(t)=x_{m}(t)-y_{m}(t)$, then we can get:

$$
\begin{aligned}
\dot{e}_{m}(t) & =-c_{m} e_{m}(t)+\sum_{z=1}^{l}\left[\varsigma_{m z}\left(x_{m}(t)\right)+\Delta \varsigma_{m z}(t)\right] f_{z}\left(x_{z}(t)\right) \\
& -\sum_{z=1}^{l}\left[\omega_{m z}\left(y_{m}(t)\right)+\Delta \omega_{m z}(t)\right] f_{z}\left(y_{z}(t)\right) \\
& +\sum_{z=1}^{l}\left[\rho_{m z}\left(x_{m}(t)\right)+\Delta \rho_{m z}(t)\right] g_{z}\left(x_{z}\left(t-\tau_{z}(t)\right)\right) \\
& -\sum_{z=1}^{l}\left[\beta_{m z}\left(y_{m}(t)\right)+\Delta \beta_{m z}(t)\right] g_{z}\left(y_{z}\left(t-\tau_{z}(t)\right)\right) \\
& -u_{m}(t), \quad t \geq 0, m=1,2, \ldots, l .
\end{aligned}
$$

To study the complete synchronization problem, some definitions on synchronization are presented below.

For two dynamical systems

$$
\left\{\begin{array}{l}
\dot{x}(t)=f(x(t)) \\
\dot{y}(t)=f(y(t))
\end{array}\right.
$$

where $x(t)=\left(x_{1}(t), x_{2}(t), \ldots, x_{l}(t)\right)^{T}, y(t)=\left(y_{1}(t), y_{2}(t)\right.$, $\left.\ldots, y_{l}(t)\right)^{T}$, and the initial conditions are $x(s)=\phi(s)=$ $\left(\phi_{1}(s), \phi_{2}(s), \ldots, \phi_{l}(s)\right)^{T}, y(s)=\varphi(s)=\left(\varphi_{1}(s), \varphi_{2}(s), \ldots\right.$, $\left.\varphi_{l}(s)\right)^{T}$, some definitions on synchronization are presented as follows.
Definition 1 [42, 43]: If there exist positive constants $Q_{1}$, $\alpha$ and $t_{0}$, such that

$$
\|x(t)-y(t)\| \leq Q_{1} \sup _{-\tau \leq s \leq 0}\|\phi(s)-\varphi(s)\| t^{-\alpha}, \quad t \geq t_{0},
$$

then two dynamical systems (7) can achieve power-rate synchronization.

Definition 2 [26-31], [35], [37]: If there exist positive constants $Q_{2}$ and $\beta$, such that

$$
\|x(t)-y(t)\| \leq Q_{2} \sup _{-\tau \leq s \leq 0}\|\phi(s)-\varphi(s)\| e^{-\beta t}, \quad t \geq 0,
$$

then two dynamical systems (7) can achieve exponential synchronization with a degree $\beta$.

When $x(t)=\left(x_{1}(t), x_{2}(t), \ldots, x_{l}(t)\right)^{T}, y(t)=\left(y_{1}(t)\right.$, $\left.y_{2}(t), \ldots, y_{l}(t)\right)^{T}$ are affected by parameter perturbations, all types of complete synchronization mentioned above are robust, that is, the corresponding robust complete synchronization can be obtained.

From the Definitions 1 and 2, power-rate synchronization [42, 43] and exponential synchronization [26-31], [35], [37] can show the process of infinite approximation and present the convergence rate. In fact, two systems can tend to same state by different convergence rates and convergence methods when time approaches to infinity. In this paper, we propose multimode function synchronization for two dynamical systems (7) to generalize these existing definitions.

Definition 3: If there exist a monotone nondecreasing continuous function $r(t)$ and a positive constant $Q$, such that

$$
\|x(t)-y(t)\| \leq Q \sup _{-\tau \leq s \leq 0}\|\phi(s)-\varphi(s)\| / r(t), \quad t \geq 0,
$$

then two dynamical systems (7) can achieve multimode function synchronization based on the convergence rate $r(t)$, where $r(s)$ is a positive constant, $s \in[-\tau, 0]$, and $\lim _{t \rightarrow+\infty} r(t)=$ $+\infty$.

When two dynamical systems (7) are written into MNN systems (5) and (4), we can have the following definition.

Definition 4: If there exist a monotone nondecreasing continuous function $r(t)$ and a positive constant $Q$, such that

$$
\|x(t)-y(t)\| \leq Q \sup _{-\tau \leq s \leq 0}\|\phi(s)-\varphi(s)\| / r(t), \quad t \geq 0,
$$

then MNN systems (5) and (4) can achieve robust multimode function synchronization based on the convergence rate $r(t)$, where $r(s)$ is a positive constant, $s \in[-\tau, 0]$, and $\lim _{t \rightarrow+\infty} r(t)=+\infty$.

By choosing different types of $r(t)$, multiple types of robust complete synchronization can be obtained. Specific multiple types of complete synchronization can be represented as follows.

Synchronization mode 1: When $r(t)$ is an exponential function, such as $r(t)=e^{\alpha t}, \alpha$ is a positive constant, system (5) and system (4) can achieve robust exponential synchronization.

Synchronization mode 2: When $r(t)$ is a polynomial function, such as $r(t)=t^{2}+1$, system (5) and system (4) can achieve robust polynomial synchronization. It is worth noting 


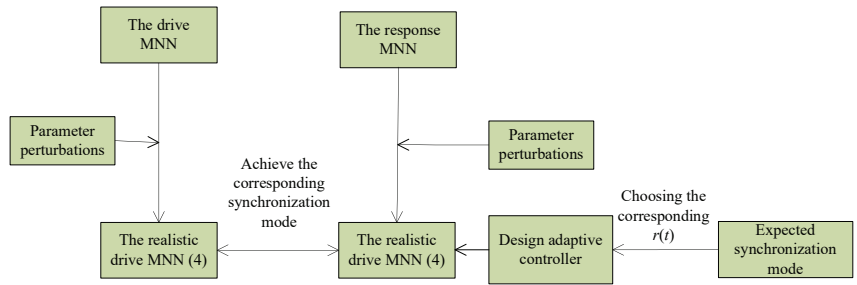

Fig. 1. The schematic diagram of robust multimode function synchronization of MNNs.

that power-rate synchronization is a special case of polynomial synchronization.

Synchronization mode 3: When $r(t)$ is a logarithmic function, such as $r(t)=\ln (t+a), a>\tau+1$, system (5) and system (4) can achieve robust logarithmical synchronization.

When $r(t)$ is different from the above-mentioned functions, we define the type of complete synchronization between system (5) and system (4) as Synchronization mode 4.

Remark 2: Definition 3 provides a general definition of multiple types of complete synchronization by mathematical expression. From Definition 3, power-rate synchronization [42, 43] and exponential synchronization [26-31], [35], [37] can be viewed as two special cases of multimode function synchronization. Therefore, multimode function synchronization is general and enlarges the existing results.

\section{Robust Multimode Function Synchronization OF MNNS WITH ADAPTIVE CONTROLLER}

The schematic diagram of robust multimode function synchronization of MNNs is shown in Fig. 1. First of all, the drive and response MNNs will change to the realistic drive and response MNNs with two types of parameter perturbations. According to some practical needs, we choose synchronization mode between the realistic drive $\mathrm{MNN}$ and the realistic response MNN. Then the corresponding function $r(t)$ and update gain $\sigma$ are chosen to design the adaptive controller, where function $r(t)$ is used to generate multiple types of robust complete synchronization including robust power-rate synchronization and robust exponential synchronization, and update gain $\sigma$ is used to adjust the speed of synchronization. Finally, the realistic drive and response MNNs can achieve the corresponding robust synchronization mode under the adaptive controller.

\section{A. Robust Multimode Function Synchronization}

In this section, we will design two adaptive controllers to achieve robust multimode function synchronization between system (4) and system (5).

Adaptive Controller (A)

Consider adaptive control (A) as follows:

$$
\left\{\begin{array}{l}
u_{m}(t)=\lambda_{m}(t) e_{m}(t)+\varepsilon_{m}(t) \operatorname{sgn}\left(e_{m}(t)\right) \\
\dot{\lambda}_{m}(t)=\theta_{m}\left[r(t) e_{m}(t)\right]^{2} \\
\dot{\varepsilon}_{m}(t)=\sigma_{m} r^{2}(t)\left|e_{m}(t)\right|
\end{array}\right.
$$

where $\theta_{m}, \sigma_{m}$ are positive constants; $\operatorname{sgn}\left(e_{m}(t)\right)$ is the sign function; $r(t)$ is a continuously differentiable and monotone nondecreasing function. $r(s), \lambda_{m}(s)$ and $\varepsilon_{m}(s)$ are positive constants, $s \in[-\tau, 0]$, and $\lim _{t \rightarrow+\infty} r(t)=+\infty, m=$ $1,2, \ldots, l$.

Assumption 1: Functions $f_{m}($.$) and g_{m}($.$) are bounded,$ namely, $\left|f_{m}(a)\right| \leq M_{m}$ and $\left|g_{m}(a)\right| \leq N_{m}$ for any $a \in \Re$, where $M_{m}$ and $N_{m}$ are positive constants; $f_{m}($.$) satisfies$ Lipschitz condition, namely, there exists constant $L_{m}>0$, so that $\left|f_{m}\left(a_{1}\right)-f_{m}\left(a_{2}\right)\right| \leq L_{m}\left|a_{1}-a_{2}\right|$ for any $a_{1}, a_{2} \in \Re$, $m=1,2, \ldots, l$.

Assumption 2: The function $r(t)$ satisfies

$$
\frac{\dot{r}(t)}{r(t)} \leq c
$$

where $c=\min _{1 \leq m \leq l}\left\{c_{m}\right\}$.

Theorem 1: MNN systems (5) and (4) can achieve robust multimode function synchronization with the adaptive controller (A) under assumptions 1 and 2.

Proof. See Appendix A.

Corollary 1: When $r(t)$ is an exponential function, such as $r(t)=e^{b t}, b>0$, MNN systems (5) and (4) can realize robust exponential synchronization with the adaptive controller (A) with no need for the assumption 2 .

Proof. See Appendix B.

Remark 3: The dynamic state coupling condition in [31] and adaptive control condition in [37] for exponential synchronization of MNNs can be seen as two special cases of adaptive controller (A) for robust multimode function synchronization of MNNs. Therefore, adaptive control method proposed herein is more general and flexible.

Corollary 2: MNN systems (5) and (4) can achieve robust multimode function synchronization under assumptions 1 and 2 with the following adaptive controller:

$$
\left\{\begin{array}{l}
u_{m}(t)=\lambda_{m}(t) e_{m}(t)+\varepsilon_{m}(t) \operatorname{sgn}\left(h_{m}\left(e_{m}(t)\right)\right) \\
\dot{\lambda}_{m}(t)=\theta_{m}\left[r(t) e_{m}(t)\right]^{2} \\
\dot{\varepsilon}_{m}(t)=\sigma_{m} r^{2}(t)\left|e_{m}(t)\right|
\end{array}\right.
$$

where $h_{m}\left(e_{m}(t)\right)=f_{m}\left(x_{m}(t)\right)-f_{m}\left(y_{m}(t)\right)$; function $f_{m}($. is strictly monotone increasing; $m=1,2, \ldots, l$.

Proof: Because $f_{m}($.$) is strictly monotone increasing, we$ can get $\operatorname{sgn}\left(h_{m}\left(e_{m}(t)\right)\right)=\operatorname{sgn}\left(e_{m}(t)\right)$, so (14) is equivalent to (12). The proof is finished.

Corollary 3: MNN systems (5) and (4) can achieve robust multimode function synchronization under assumptions 1 and 2 with the following adaptive controller:

$$
\left\{\begin{array}{l}
u_{m}(t)=\lambda_{m}(t) e_{m}(t)-\varepsilon_{m}(t) \operatorname{sgn}\left(h_{m}\left(e_{m}(t)\right)\right) \\
\dot{\lambda}_{m}(t)=\theta_{m}\left[r(t) e_{m}(t)\right]^{2} \\
\dot{\varepsilon}_{m}(t)=\sigma_{m} r^{2}(t)\left|e_{m}(t)\right|
\end{array}\right.
$$

where $h_{m}\left(e_{m}(t)\right)=f_{m}\left(x_{m}(t)\right)-f_{m}\left(y_{m}(t)\right)$; function $f_{m}($. is strictly monotone decreasing; $m=1,2, \ldots, l$.

Adaptive Controller (B)

Next, we design the other adaptive controller. We consider adaptive control (B) as follows:

$$
\left\{\begin{array}{l}
u_{m}(t)=\lambda_{m}(t) h_{m}\left(e_{m}(t)\right)+\varepsilon_{m}(t) \operatorname{sgn}\left(e_{m}(t)\right) \\
\dot{\lambda}_{m}(t)=\theta_{m} r^{2}(t) e_{m}(t) h_{m}\left(e_{m}(t)\right) \\
\dot{\varepsilon}_{m}(t)=\sigma_{m} r^{2}(t)\left|e_{m}(t)\right|
\end{array}\right.
$$


where $\theta_{m}, \sigma_{m}$ are positive constants; $\operatorname{sgn}\left(e_{m}(t)\right)$ is the sign function; $r(t)$ is a continuously differentiable and monotone nondecreasing function. $r(s), \lambda_{m}(s)$ and $\varepsilon_{m}(s)$ are positive constants, $s \in[-\tau, 0]$, and $\lim _{t \rightarrow+\infty} r(t)=+\infty, m=$ $1,2, \ldots, l$.

Assumption 3: The transmission delay $\tau_{m}(t), m=$ $1,2, \ldots, l$, satisfies

$$
\dot{\tau}_{m}(t) \leq 1
$$

We change assumption 1 to assumption 4 which will be used in Theorem 2.

Assumption 4: Functions $f_{m}($.$) and g_{m}($.$) are bounded,$ namely, $\left|f_{m}(a)\right| \leq M_{m}$ and $\left|g_{m}(a)\right| \leq N_{m}$ for any $a \in \Re$, where $M_{m}$ and $N_{m}$ are positive constants; and for any $a_{1}, a_{2} \in \Re$, there exists constant $L_{m}>0$, such that

$$
0 \leq \frac{f_{m}\left(a_{1}\right)-f_{m}\left(a_{2}\right)}{a_{1}-a_{2}} \leq L_{m}
$$

$m=1,2, \ldots, l$.

Theorem 2: MNN systems (5) and (4) can realize robust multimode function synchronization under assumptions 2, 3 and 4 with the adaptive controller (B).

Proof. See Appendix C.

Remark 4: In some research results, such as [26, 27], $[31,32]$ and [35], the activation function $g_{m}($.$) needs to satisfy$ Lipschitz condition to achieve synchronization. In this paper, it is not necessary for $g_{m}($.$) to satisfy Lipschitz condition$ by utilizing adaptive controller (A) or (B) to synchronize MNNs. Therefore, the conditions herein are less conservative, compared with the above-mentioned research results.

From assumption 4, we can get the activation functions $f_{m}($.$) is monotone nondecreasing. So, when the function f_{m}($. is strictly monotone increasing, the following corollary can be obtained.

Corollary 4: MNN systems (5) and (4) can realize robust multimode function synchronization under assumptions 2, 3 and 4 with the following adaptive controller:

$$
\left\{\begin{array}{l}
u_{m}(t)=\lambda_{m}(t) h_{m}\left(e_{m}(t)\right)+\varepsilon_{m}(t) \operatorname{sgn}\left(h_{m}\left(e_{m}(t)\right)\right) \\
\dot{\lambda}_{m}(t)=\theta_{m} r^{2}(t) e_{m}(t) h_{m}\left(e_{m}(t)\right) \\
\dot{\varepsilon}_{m}(t)=\sigma_{m} r^{2}(t)\left|e_{m}(t)\right|
\end{array}\right.
$$

where $m=1,2, \ldots, l$.

Remark 5: Adaptive controller (A) consists of $\lambda_{m}(t) e_{m}(t)$ and $\varepsilon_{m}(t) \operatorname{sgn}\left(e_{m}(t)\right)$. As time $t \rightarrow \infty, e_{m}(t) \rightarrow 0$, $\operatorname{sgn}\left(e_{m}(t)\right)=1$ or -1 (if $e_{m}(t) \neq 0$ ). Thus update gain $\sigma_{m}$ makes the decisive factor for controlling the synchronization speed. In other words, synchronization speed increases with $\sigma$. The similar results can be obtained for adaptive controller (B).

Remark 6: In [26], it is very hard to get the solutions of the conditions for Theorem 1 because a gain matrix needs to be chosen such that Hamiltonian matrix has no eigenvalues on the imaginary axis. It needs use MATLAB Tool-box to solve this problem. In [27], [35], [39], [41], linear matrix inequality (LMI) is applied to find some matrices satisfying the conditions of Theorems. Some constants need to be chosen such that some LMI conditions of Theorems are satisfied in [40]. To some extent, these conditions of Theorems in [26, 27],

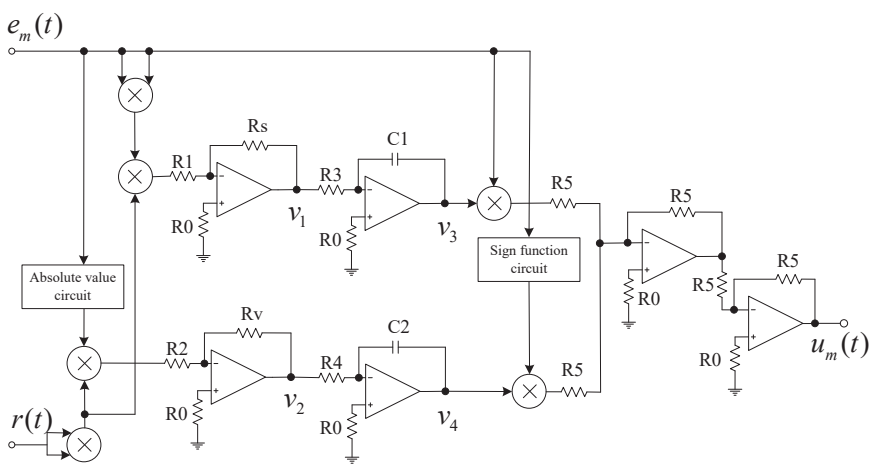

Fig. 2. The circuit design of adaptive controller (A).

[35], [39-41] are complex to find the solutions, and they can only be solved by users via some computer-aided tools. In this paper, it is not necessary to find the solutions for the conditions of Theorems 1 and 2. According to practical needs, users choose synchronization mode between the realistic drive MNN (4) and the realistic response MNN (5). Then the corresponding function $r(t)$ and update gain $\sigma$ are chosen to design the adaptive controller. Finally, the realistic drive and response MNNs can achieve the corresponding robust synchronization mode under the adaptive controller and some assumptions.

Remark 7: In some recent published work [42-46], powerrate synchronization $[42,43]$ and exponential synchronization [44-46] were addressed. Compared with these results [4246], the proposed robust multimode function synchronization has great theoretical and practical significance because of its strong anti-jamming ability, and can generalize the existing definitions of multiple types of complete synchronization due to its being able to achieve multiple types of robust complete synchronization (that is multiple convergence methods). The robust multimode function synchronization proposed in this paper has three remarkable features: 1) Robustness - Under two types of parameter perturbations, MNNs can achieve complete synchronization; 2) Fast convergence rate - Choose an appropriate update gain $\sigma$ according to the practical needs, the fast convergence rate can be obtained; 3) Multiple types of complete synchronization - Function $r(t)$ can be freely chosen, such as exponential function, polynomial function, and logarithmical function, thus corresponding robust complete synchronization can be obtained.

\section{B. Circuit Design and Complexity Analysis of Adaptive Con- troller}

The designed circuit of adaptive controller (A) is shown in Fig. 2.

The absolute value circuit and sign function circuit in Fig. 2 can change $e_{m}(t)$ into $\left|e_{m}(t)\right|$ and $\operatorname{sgn}\left(e_{m}(t)\right)$, respectively. From Fig. 2, we can write voltage $v_{1}$ and $v_{2}$, given by

$$
v_{1}=-\frac{R s}{R 1} e_{m}^{2}(t) r^{2}(t)
$$

and

$$
v_{2}=-\frac{R v}{R 2} r^{2}(t)\left|e_{m}(t)\right|
$$


We can also derive voltage $v_{3}$ and $v_{4}$ as

$$
\begin{gathered}
\dot{v}_{3}=-\frac{v_{1}}{C 1 R 3}=\frac{R s}{C 1 R 3 R 1} e_{m}^{2}(t) r^{2}(t), \\
\dot{v}_{4}=-\frac{v_{2}}{C 2 R 4}=\frac{R v}{C 2 R 4 R 2} r^{2}(t)\left|e_{m}(t)\right| .
\end{gathered}
$$

Note that the output voltage $u_{m}(t)$ can be given by

$$
u_{m}(t)=v_{3} e_{m}(t)+v_{4} \operatorname{sgn}\left(e_{m}(t)\right) .
$$

Setting $\frac{R s}{C 1 R 3 R 1}=\theta_{m}$ and $\frac{R v}{C 2 R 4 R 2}=\sigma_{m}$, then $v_{3}, v_{4}$ and $u_{m}(t)$ constitute the adaptive controller (A).

Similarly, the circuit of adaptive controller (B) can also be designed, which is not detailed here.

Now, we discuss the complexity of adaptive controllers. In the following, we mainly analyze the time complexity of adaptive controller (A), but similar analysis can also be conducted for adaptive controller (B). For adaptive controller (A), the derivative of $\lambda_{m}(t)$ needs four multiplication operations. The derivative of $\varepsilon_{m}(t)$ needs three multiplication operations and one absolute value operation. In addition, $u_{m}(t)$ needs two multiplication operations, one addition operation and one symbolic function operation. Therefore, the adaptive controller (A) requires 12 operations in total. We assume the step length is $h$ second, and the runtime of system is $n$ seconds. Then the time complexity of adaptive controller (A) is $T(n)=$ $O(12 n / h)=O(n)$. In the same way, we can also get the time complexity of adaptive controller (B), which is given by $T(n)=O(n)$.

Remark 8: In [33, 34], [41] and [44], synchronization of MNNs was addressed by linear feedback control. However, the control gain of the linear feedback controller must be maximal, which means that there exists waste to some extent in practical applications. Different from the linear feedback controller, the proposed adaptive controller can increase the control gain on the basis of adaptive methods and thus is more flexible. Computationally, the adaptive controller is efficient and suitable for synchronization of MNNs due to its low time complexity. Our analytical derivations in the above and numerical examples to be presented in the following demonstrate the feasibility of the adaptive controller for the synchronization of MNNs theoretically. Furthermore, our circuit design in the above proves the feasibility of the practical implementation of the adaptive controller.

\section{NUMERICAL EXAMPLES}

The above theoretical results and analysis can be verified in this section.

Example 1: Consider a two-neuron drive MNN as follows:

$$
\begin{aligned}
& \dot{x}_{m}(t)=-c_{m} x_{m}(t)+\sum_{z=1}^{2}\left[\varsigma_{m z}\left(x_{m}(t)\right)+\Delta \varsigma_{m z}(t)\right] f_{z}\left(x_{z}(t)\right) \\
& +\sum_{z=1}^{2}\left[\rho_{m z}\left(x_{m}(t)\right)+\Delta \rho_{m z}(t)\right] g_{z}\left(x_{z}\left(t-\tau_{z}(t)\right)\right) \\
& +I_{m}(t), \quad m=1,2
\end{aligned}
$$

where $c_{m}=1 ; \tau_{m}(t)=\left(e^{t}-1\right) /\left(e^{t}+1\right) ; \tau=1 ; \dot{\tau}_{m}(t) \leq 0.5$; activation function $f_{z}(w)=g_{z}(w)=\tanh (w) ; \kappa_{m}=1$; and $I_{m}(t)=0 ; m, z=1,2$.

$$
\begin{gathered}
\varsigma_{11}\left(x_{1}(t)\right)= \begin{cases}1.95, & \Gamma_{1}, \\
1.26, & \Gamma_{2},\end{cases} \\
\varsigma_{12}\left(x_{1}(t)\right)= \begin{cases}-0.1, & \Gamma_{1}, \\
-0.2, & \Gamma_{2},\end{cases} \\
\varsigma_{21}\left(x_{2}(t)\right)= \begin{cases}-4.9, & \Gamma_{1}, \\
-5.0, & \Gamma_{2},\end{cases} \\
\varsigma_{22}\left(x_{2}(t)\right)= \begin{cases}2.9, & \Gamma_{1}, \\
2.32, & \Gamma_{2},\end{cases} \\
\rho_{11}\left(x_{1}(t)\right)= \begin{cases}-1.48, & \Gamma_{1}, \\
-1.5, & \Gamma_{2},\end{cases} \\
\rho_{12}\left(x_{1}(t)\right)= \begin{cases}-0.1, & \Gamma_{1}, \\
-2.09, & \Gamma_{2},\end{cases} \\
\rho_{21}\left(x_{2}(t)\right)=\left\{\begin{array}{ll}
-0.12, & \Gamma_{1}, \\
-0.5, & \Gamma_{2},
\end{array},\right. \\
\rho_{22}\left(x_{2}(t)\right)= \begin{cases}-2.2, & \Gamma_{1}, \\
-1.8, & \Gamma_{2} .\end{cases}
\end{gathered}
$$

Uncertain parameters are

$$
\begin{aligned}
& {\left[\begin{array}{cc}
\Delta \varsigma_{11}(t) & \Delta \varsigma_{12}(t) \\
\Delta \varsigma_{21}(t) & \Delta \varsigma_{22}(t)
\end{array}\right]=} \\
& {\left[\begin{array}{cc}
3 \times 10^{-2} \sin (t) & 1 \times 10^{-2} \cos (t) \\
5 \times 10^{-2} \tanh (t) & 0
\end{array}\right],} \\
& {\left[\begin{array}{cc}
\Delta \rho_{11}(t) & \Delta \rho_{12}(t) \\
\Delta \rho_{21}(t) & \Delta \rho_{22}(t)
\end{array}\right]=} \\
& {\left[\begin{array}{cc}
1 \times 10^{-2} \sin (t) & 0 \\
1 \times 10^{-2} \cos (t) & 2 \times 10^{-2} \sin (t)
\end{array}\right] .}
\end{aligned}
$$

Consider the response MNN as follows:

$$
\begin{aligned}
& \dot{y}_{m}(t)=-c_{m} y_{m}(t)+\sum_{z=1}^{2}\left[\omega_{m z}\left(y_{m}(t)\right)+\Delta \omega_{m z}(t)\right] f_{z}\left(y_{z}(t)\right) \\
& +\sum_{z=1}^{2}\left[\beta_{m z}\left(y_{m}(t)\right)+\Delta \beta_{m z}(t)\right] g_{z}\left(y_{z}\left(t-\tau_{z}(t)\right)\right) \\
& +I_{m}(t)+u_{m}(t), \quad m=1,2,
\end{aligned}
$$

where

$$
\begin{aligned}
& \omega_{11}\left(y_{1}(t)\right)= \begin{cases}2.02, & \Omega_{1}, \\
1.21, & \Omega_{2},\end{cases} \\
& \omega_{12}\left(y_{1}(t)\right)= \begin{cases}-0.13, & \Omega_{1}, \\
-0.22, & \Omega_{2},\end{cases} \\
& \omega_{21}\left(y_{2}(t)\right)= \begin{cases}-4.82, & \Omega_{1}, \\
-5.1, & \Omega_{2},\end{cases} \\
& \omega_{22}\left(y_{2}(t)\right)= \begin{cases}3.06, & \Omega_{1}, \\
2.1, & \Omega_{2},\end{cases} \\
& \beta_{11}\left(y_{1}(t)\right)= \begin{cases}-1.54, & \Omega_{1}, \\
-1.59, & \Omega_{2},\end{cases} \\
& \beta_{12}\left(y_{1}(t)\right)= \begin{cases}-0.11, & \Omega_{1}, \\
-0.06, & \Omega_{2},\end{cases}
\end{aligned}
$$




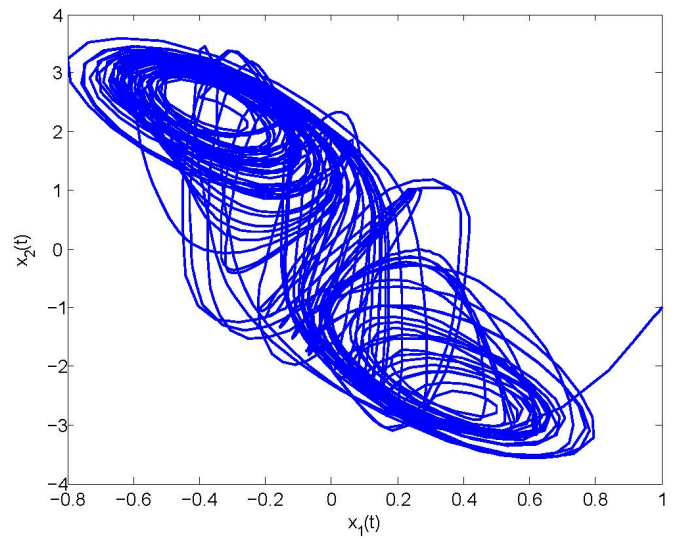

Fig. 3. Phase plot between $x_{1}(t)$ and $x_{2}(t)$ of system (22).

$$
\begin{aligned}
& \beta_{21}\left(y_{2}(t)\right)= \begin{cases}-0.29, & \Omega_{1}, \\
-0.21, & \Omega_{2},\end{cases} \\
& \beta_{22}\left(y_{2}(t)\right)= \begin{cases}-2.52, & \Omega_{1}, \\
-2.44, & \Omega_{2} .\end{cases}
\end{aligned}
$$

Uncertain parameters are

$$
\begin{aligned}
& {\left[\begin{array}{ll}
\Delta \omega_{11}(t) & \Delta \omega_{12}(t) \\
\Delta \omega_{21}(t) & \Delta \omega_{22}(t)
\end{array}\right]=} \\
& {\left[\begin{array}{cc}
1 \times 10^{-2} \cos (t) & 3 \times 10^{-2} \sin (t) \\
0 & 1 \times 10^{-2} \tanh (t)
\end{array}\right],} \\
& {\left[\begin{array}{cc}
\Delta \beta_{11}(t) & \Delta \beta_{12}(t) \\
\Delta \beta_{21}(t) & \Delta \beta_{22}(t)
\end{array}\right]=} \\
& {\left[\begin{array}{cc}
0 & 4 \times 10^{-2} \cos (t) \\
6 \times 10^{-2} \tanh (t) & 1.5 \times 10^{-2} \sin (t)
\end{array}\right] .}
\end{aligned}
$$

The other parameters are the same as (19).

When initial conditions are $\left(x_{1}(s), x_{2}(s)\right)^{T}=(1,-1)^{T}$ for $s \in[-1,0]$, the phase plot between $x_{1}(t)$ and $x_{2}(t)$ of system (19) is shown in Fig. 3. The MNN system (19) exhibits chaotic behavior with the above initial conditions. When the initial conditions of system (20) are $\left(y_{1}(s), y_{2}(s)\right)^{T}=(-0.6,1.2)^{T}$, $s \in[-1,0]$, the state trajectories of $x_{1}(t)$ and $y_{1}(t), x_{2}(t)$ and $y_{2}(t)$ without feedback controller are shown in Fig. 4.

We consider control input $u_{m}(t)$ as adaptive controller (A), and set $r(t)=\left(t^{2}+1\right)$ and initial values $\left(\lambda_{1}(s), \lambda_{2}(s)\right)^{T}=$ $(0,0)^{T},\left(\varepsilon_{1}(s), \varepsilon_{2}(s)\right)^{T}=(0,0)^{T}$ for $s \in[-1,0]$. To better explore how $\sigma_{m}$ will affect the synchronization speed, we take into consideration three cases: 1) $\theta_{m}=1, \sigma_{m}=5$;2) $\theta_{m}=1$, $\sigma_{m}=0.5$; 3) $\theta_{m}=1, \sigma_{m}=0.05 ; m=1,2$. Fig. 5 presents errors $e_{1}(t)$ and $e_{2}(t)$ with 10 arbitrary initial conditions. The 10 arbitrary initial conditions satisfy $x_{m}(s)=\phi_{m}(s) \in$ $[-1.8,1.8]$ and $y_{m}(s)=\varphi_{m}(s) \in[-1.8,1.8]$ for $s \in[-1,0]$, $m=1,2$. Thus, the initial errors $e_{m}(s) \in[-3.6,3.6]$, $s \in[-1,0]$. From Fig. 5, MNN systems (20) and (19) can realize robust polynomial synchronization under adaptive controller (A). Moreover, synchronization speed increases with $\sigma$.

Fig. 6 shows the relationship between convergence time of error $e_{m}(t)$ and parameter $\sigma_{m}$. It can be shown that synchronization speed increases with $\sigma$. When parameter $\sigma$ is less than 10, synchronization speed is almost proportional
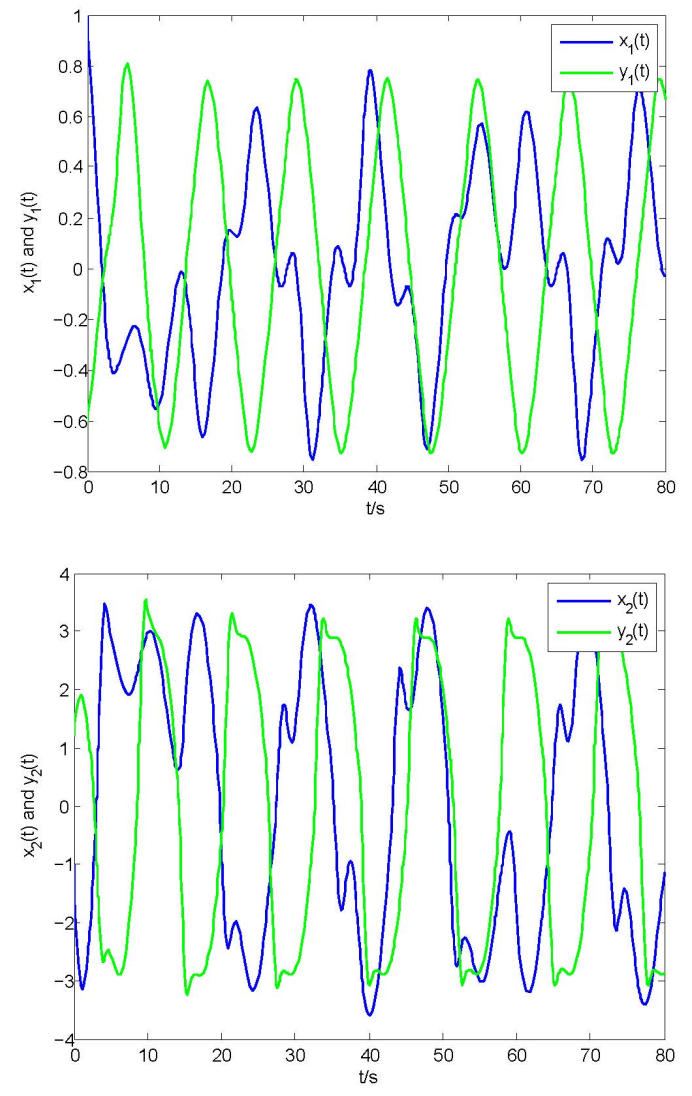

Fig. 4. State trajectories of $x_{1}(t)$ and $y_{1}(t), x_{2}(t)$ and $y_{2}(t)$ without feedback controller.

to the logarithm of $\sigma$. When parameter $\sigma$ is greater than 10 , convergence time is very small and synchronization speed is not proportional to the logarithm of $\sigma$. Convergence time of error $e_{m}(t)$ is close to 0 with parameter $\sigma_{m}=10^{2}$. Theoretically, convergence time of error $e_{m}(t)$ can infinitely close to 0 along with parameter $\sigma_{m}$. However, the larger the parameter $\sigma$, the greater the computation and storage. Therefore, fast synchronization speed can be obtained under small amount of computation and storage when parameter $\sigma$ is in the interval $\left[10^{0}, 10^{2}\right]$. In practical applications, the upper bound for parameter $\sigma_{m}$ should be $10^{2}$.

Example 2: Consider another two-neuron drive MNN as follows:

$$
\begin{aligned}
& \dot{x}_{m}(t)=-c_{m} x_{m}(t)+\sum_{z=1}^{2}\left[\varsigma_{m z}\left(x_{m}(t)\right)+\Delta \varsigma_{m z}(t)\right] f_{z}\left(x_{z}(t)\right) \\
& +\sum_{z=1}^{2}\left[\rho_{m z}\left(x_{m}(t)\right)+\Delta \rho_{m z}(t)\right] g_{z}\left(x_{z}\left(t-\tau_{z}(t)\right)\right) \\
& +I_{m}(t), \quad m=1,2,
\end{aligned}
$$

where $c_{m}=1 ; \tau_{m}(t)=\left(e^{t}-1\right) /\left(e^{t}+1\right) ; \tau=1 ; \dot{\tau}_{m}(t) \leq 0.5$; activation function $f_{z}(w)=g_{z}(w)=\frac{|w+1|-|w-1|}{2} ; \kappa_{m}=1$; and $I_{m}(t)=0 ; m, z=1,2$.

Uncertain parameters are

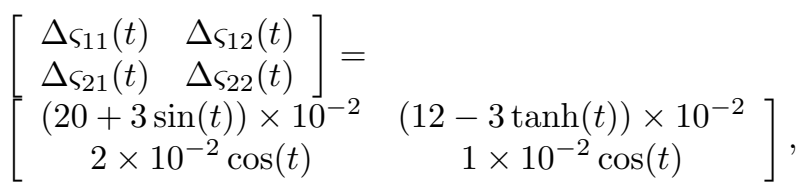



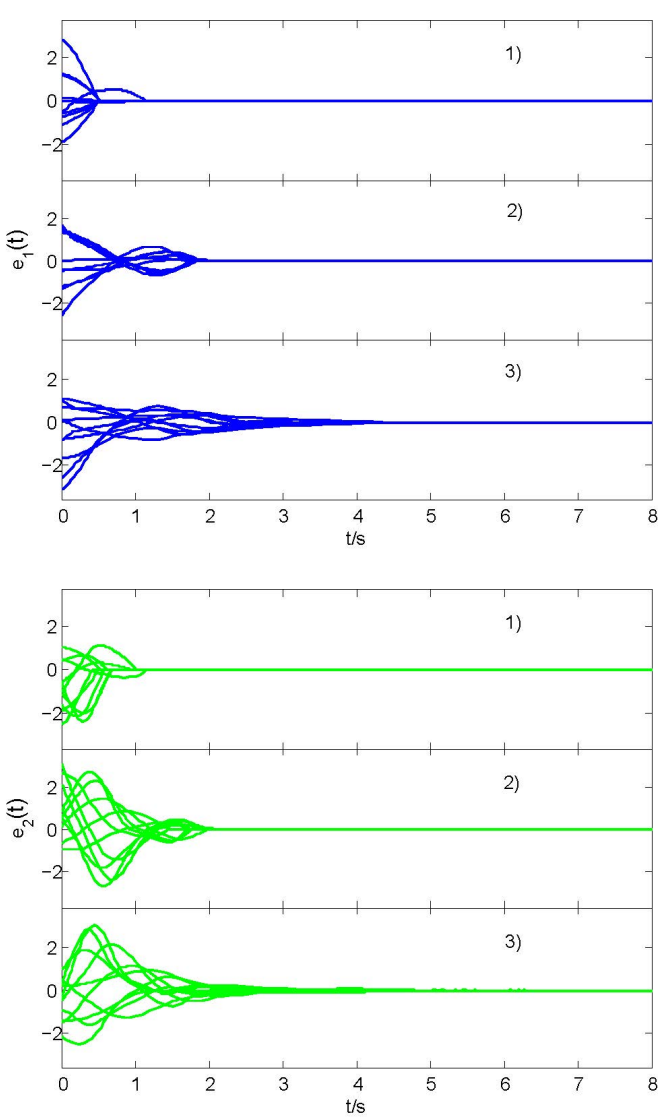

Fig. 5. Synchronization errors $e_{1}(t)$ and $e_{2}(t)$ with 10 arbitrary initial conditions using adaptive controller (A) for three cases: 1) $\theta_{m}=1, \sigma_{m}=5$; 2) $\theta_{m}=1, \sigma_{m}=0.5$; 3) $\theta_{m}=1, \sigma_{m}=0.05 ; m=1$, 2 . It is obtained that synchronization speed increases with $\sigma$.

$$
\begin{aligned}
& {\left[\begin{array}{cc}
\Delta \rho_{11}(t) & \Delta \rho_{12}(t) \\
\Delta \rho_{21}(t) & \Delta \rho_{22}(t)
\end{array}\right]=} \\
& {\left[\begin{array}{cc}
2 \times 10^{-2} \tanh (t) & (20+3 \cos (t)) \times 10^{-2} \\
3 \times 10^{-2} \sin (t) & (1+2 \sin (t)) \times 10^{-2}
\end{array}\right] .}
\end{aligned}
$$

The other parameters are the same as (19).

Set the response MNN as follows:

$$
\begin{aligned}
& \dot{y}_{m}(t)=-c_{m} y_{m}(t)+\sum_{z=1}^{2}\left[\omega_{m z}\left(y_{m}(t)\right)+\Delta \omega_{m z}(t)\right] f_{z}\left(y_{z}(t)\right) \\
& +\sum_{z=1}^{2}\left[\beta_{m z}\left(y_{m}(t)\right)+\Delta \beta_{m z}(t)\right] g_{z}\left(y_{z}\left(t-\tau_{z}(t)\right)\right) \\
& +I_{m}(t)+u_{m}(t), \quad m=1,2 .
\end{aligned}
$$

Uncertain parameters are

$$
\begin{aligned}
& {\left[\begin{array}{ll}
\Delta \omega_{11}(t) & \Delta \omega_{12}(t) \\
\Delta \omega_{21}(t) & \Delta \omega_{22}(t)
\end{array}\right]=} \\
& {\left[\begin{array}{cc}
(3-\tanh (t)) \times 10^{-2} & 2 \times 10^{-2} \cos (t) \\
4 \times 10^{-2} \sin (t) & (2+\sin (t)) \times 10^{-2}
\end{array}\right],} \\
& {\left[\begin{array}{cc}
\Delta \beta_{11}(t) & \Delta \beta_{12}(t) \\
\Delta \beta_{21}(t) & \Delta \beta_{22}(t)
\end{array}\right]=} \\
& {\left[\begin{array}{cc}
(1+3 \sin (t)) \times 10^{-2} & 2 \times 10^{-2} \tanh (t) \\
(2-\sin (t)) \times 10^{-2} & 2 \times 10^{-2} \cos (t)
\end{array}\right] .}
\end{aligned}
$$

The other parameters are the same as (20).

We consider control input $u_{m}(t)$ as adaptive controller (B) and initial values $\left(\lambda_{1}(s), \lambda_{2}(s)\right)^{T}=(0,0)^{T}$,
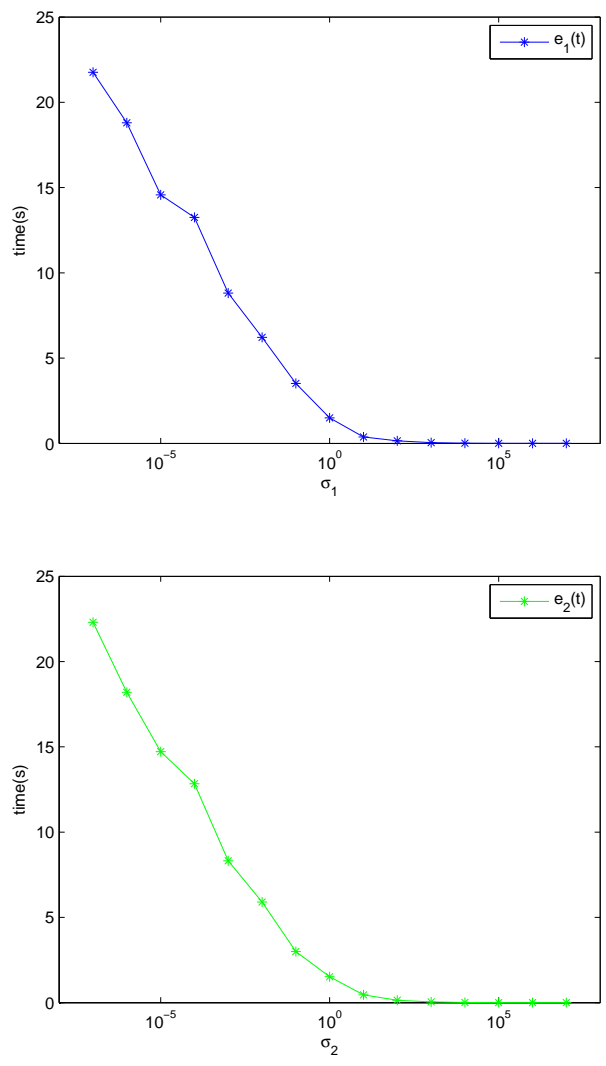

Fig. 6. The relationship between convergence time of error $e_{m}(t)$ and parameter $\sigma_{m}$.

$\left(\varepsilon_{1}(s), \varepsilon_{2}(s)\right)^{T}=(0,0)^{T}$ for $s \in[-1,0]$. Set $r(t)=e^{0.3 t}$, $\theta_{m}=1, \sigma_{m}=2, m=1,2$. When the initial conditions are $\left(x_{1}(s), x_{2}(s)\right)^{T}=(0.8,0.3)^{T},\left(y_{1}(s), y_{2}(s)\right)^{T}=$ $(-0.2,0.1)^{T}, s \in[-1,0]$, the state trajectories of $x_{1}(t)$ and $y_{1}(t), x_{2}(t)$ and $y_{2}(t)$ without feedback controller and with adaptive controller (B) are shown in Fig. 7 and Fig. 8, respectively.

This paper considers three cases: 1) $\theta_{m}=1, \sigma_{m}=20$;2) $\theta_{m}=1, \sigma_{m}=2$; 3) $\theta_{m}=1, \sigma_{m}=0.2 ; m=1$, 2. Fig. 9 presents the corresponding errors $e_{1}(t)$ and $e_{2}(t)$ with another 10 arbitrary initial conditions. From Fig. 9 and Definition 4, we can get that MNN systems (22) and (21) can realize robust exponential synchronization using adaptive controller (B), and synchronization speed increases with $\sigma$.

Fig. 10 shows the relationship between convergence time of error $e_{m}(t)$ and parameter $\sigma_{m}$. It can be shown that synchronization speed increases with $\sigma$. Similar to example 1, when parameter $\sigma$ is in the interval $\left[10^{0}, 10^{2}\right]$, fast synchronization speed can be obtained under small amount of computation and storage. In practical applications, the upper bound for parameter $\sigma_{m}$ should be $10^{2}$.

\section{CONCLUSION}

In this paper, a general definition of multiple types of complete synchronization by mathematical expression, that is, multimode function synchronization is proposed for the first time. Compared with other types of complete synchro- 

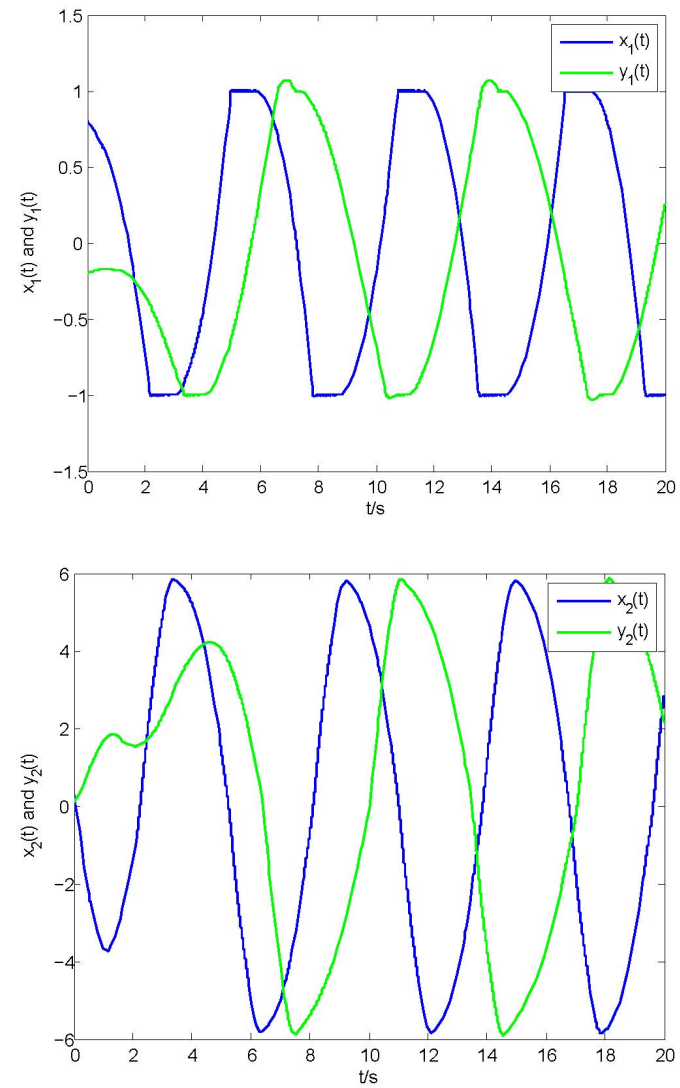

Fig. 7. State trajectories of $x_{1}(t)$ and $y_{1}(t), x_{2}(t)$ and $y_{2}(t)$ without feedback controller

nization such as power-rate synchronization and exponential synchronization, multimode function synchronization has its own unique features and advantages. (1) Multimode function synchronization can generalize the existing definitions of multiple types of complete synchronization. (2) Multimode function synchronization can generate multiple types of complete synchronization including power-rate synchronization and exponential synchronization by choosing different types of $r(t)$. This is unique feature of the proposed approach, which power-rate synchronization and exponential synchronization do not possess. (3) It is convenient to observe the process of infinite approximation and present different convergence rate when two systems tend to same state as time goes on. Therefore, the obtained results are general and enlarge the existing results. Then, two adaptive controllers are designed to achieve robust multimode function synchronization between the drive and response MNN systems with parameter perturbations and time-varying delays.

However, there are two disadvantages in the proposed method. Firstly, synchronization speed is not proportional to the logarithm of update gain $\sigma$ when it is larger than $10^{2}$. In other words, when update gain $\sigma$ is large enough, the change of convergence rate is limited. Secondly, for the type of complete synchronization to be achieved, we need to set the corresponding function $r(t)$ in advance. We will continue to solve these problems in our further research. In the future research, multimode function synchronization can be further
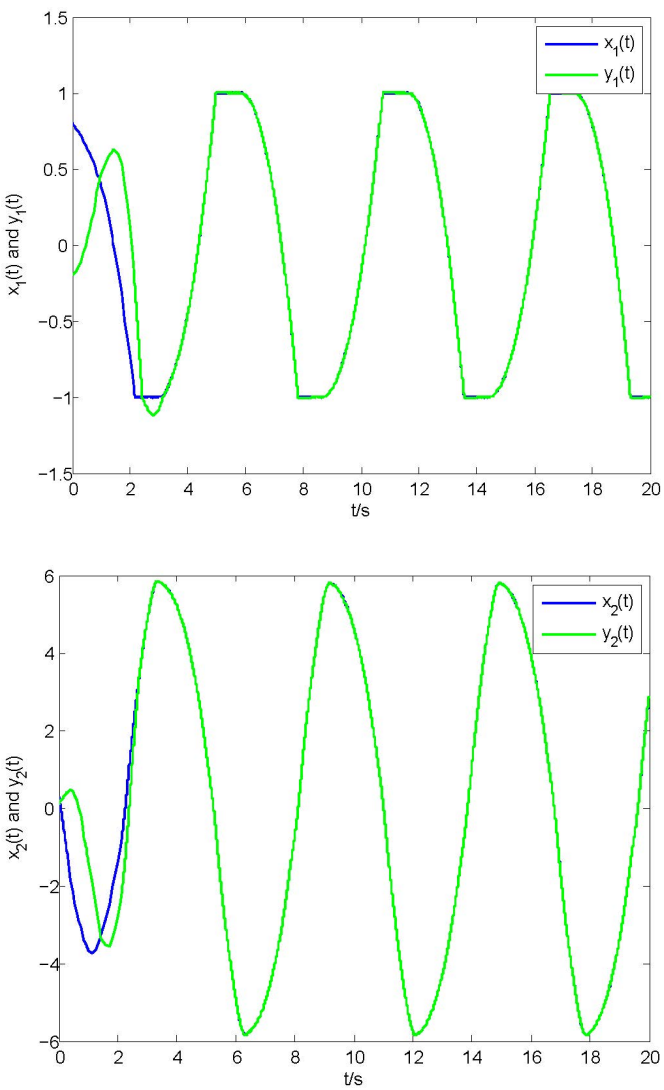

Fig. 8. State trajectories of $x_{1}(t)$ and $y_{1}(t), x_{2}(t)$ and $y_{2}(t)$ using adaptive controller (B).

developed in different systems, such as chaotic networks systems, inertial MNNs, coupled dynamical systems. Moreover, it can also be used in dynamical systems with multiple stable equilibrium states [21-23]. From the application point of view, multimode function synchronization can be applied in some fields $[15,16]$, such as information processing and secure communication. Therefore, the proposed synchronization has important theoretical value and practical significance.

\section{APPENDIX}

\section{A. Proof of Theorem 1}

Proof. Consider a Lyapunov function as:

$$
\begin{aligned}
V_{1}(t) & =\frac{1}{2} \sum_{m=1}^{l}\left[r(t) e_{m}(t)\right]^{2}+\sum_{m=1}^{l} \frac{1}{2 \theta_{m}} \lambda_{m}^{2}(t) \\
& +\sum_{m=1}^{l} \frac{1}{2 \sigma_{m}}\left(\varepsilon_{m}(t)-\Theta_{m}\right)^{2}
\end{aligned}
$$

where $\Theta_{m}$ is positive constant that will be decided.

The derivative of $V_{1}(t)$ can be written as: 

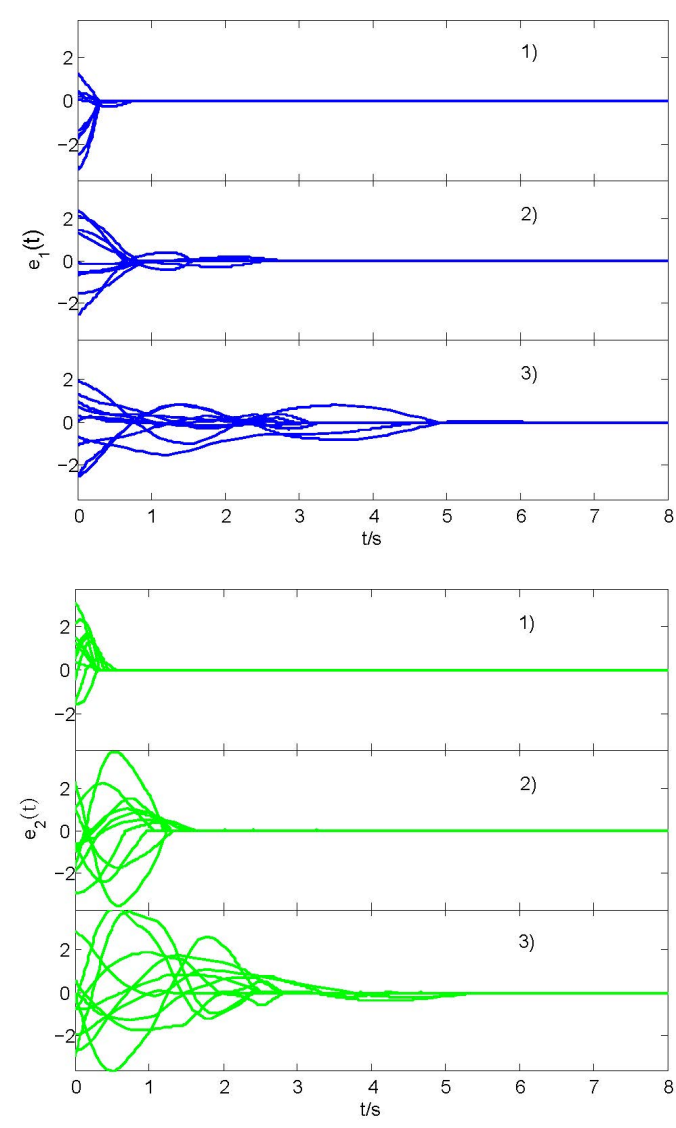

Fig. 9. Synchronization errors $e_{1}(t)$ and $e_{2}(t)$ with 10 arbitrary initial conditions using adaptive controller (B) for three cases: 1) $\theta_{m}=1, \sigma_{m}=20$; 2) $\theta_{m}=1, \sigma_{m}=2$; 3) $\theta_{m}=1, \sigma_{m}=0.2 ; m=1,2$. It is obtained that synchronization speed increases with $\sigma$.

$$
\begin{aligned}
& \dot{V}_{1}(t)=\sum_{m=1}^{l} r(t) \dot{r}(t) e_{m}^{2}(t)+\sum_{m=1}^{l} r^{2}(t) e_{m}(t)\left\{-c_{m} e_{m}(t)\right. \\
& +\sum_{z=1}^{l}\left[\varsigma_{m z}\left(x_{m}(t)\right)+\Delta \varsigma_{m z}(t)\right] f_{z}\left(x_{z}(t)\right) \\
& -\sum_{z=1}^{l}\left[\omega_{m z}\left(y_{m}(t)\right)+\Delta \omega_{m z}(t)\right] f_{z}\left(y_{z}(t)\right) \\
& +\sum_{z=1}^{l}\left[\rho_{m z}\left(x_{m}(t)\right)+\Delta \rho_{m z}(t)\right] g_{z}\left(x_{z}\left(t-\tau_{z}(t)\right)\right) \\
& -\sum_{z=1}^{l}\left[\beta_{m z}\left(y_{m}(t)\right)+\Delta \beta_{m z}(t)\right] g_{z}\left(y_{z}\left(t-\tau_{z}(t)\right)\right) \\
& \left.-\lambda_{m}(t) e_{m}(t)-\varepsilon_{m}(t) \operatorname{sgn}\left(e_{m}(t)\right)\right\} \\
& +\sum_{m=1}^{l} \lambda_{m}(t)\left[r(t) e_{m}(t)\right]^{2}+\sum_{m=1}^{l}\left(\varepsilon_{m}(t)-\Theta_{m}\right) r^{2}(t)\left|e_{m}(t)\right| \\
& =\sum_{m=1}^{l} r(t) \dot{r}(t) e_{m}^{2}(t)+\sum_{m=1}^{l} r^{2}(t) e_{m}(t)\left\{-c_{m} e_{m}(t)\right. \\
& +\sum_{z=1}^{l}\left[\varsigma_{m z}\left(x_{m}(t)\right)+\Delta \varsigma_{m z}(t)\right] f_{z}\left(x_{z}(t)\right) \\
& -\sum_{z=1}^{l}\left[\omega_{m z}\left(y_{m}(t)\right)+\Delta \omega_{m z}(t)\right] f_{z}\left(y_{z}(t)\right) \\
& +\sum_{z=1}^{l}\left[\rho_{m z}\left(x_{m}(t)\right)+\Delta \rho_{m z}(t)\right] g_{z}\left(x_{z}\left(t-\tau_{z}(t)\right)\right) \\
& \left.-\sum_{z=1}^{l}\left[\beta_{m z}\left(y_{m}(t)\right)+\Delta \beta_{m z}(t)\right] g_{z}\left(y_{z}\left(t-\tau_{z}(t)\right)\right)\right\} \\
& -\sum_{m=1}^{l} \Theta_{m} r^{2}(t)\left|e_{m}(t)\right| . \\
& \\
& +{ }_{m}(t)
\end{aligned}
$$
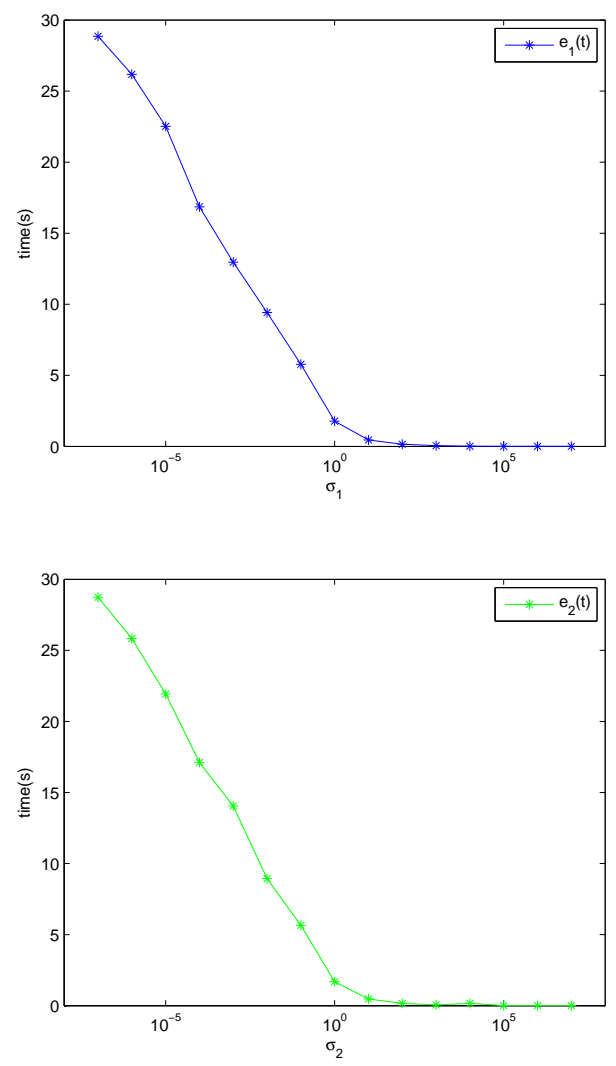

Fig. 10. The relationship between convergence time of error $e_{m}(t)$ and parameter $\sigma_{m}$.

Uncertain parameters $\Delta \varsigma_{m z}(t), \Delta \rho_{m z}(t), \Delta \omega_{m z}(t)$ and $\Delta \beta_{m z}(t)$ are bounded, namely, $\left|\Delta \varsigma_{m z}(t)\right| \leq \mu_{m z}$, $\left|\Delta \rho_{m z}(t)\right| \leq v_{m z},\left|\Delta \omega_{m z}(t)\right| \leq \chi_{m z},\left|\Delta \beta_{m z}(t)\right| \leq$ $\Upsilon_{m z}$, where $\mu_{m z}, v_{m z}, \chi_{m z}$ and $\Upsilon_{m z}$ are positive constants. Moreover, $\hat{\varsigma}_{m z}=\max \left\{\left|\dot{\varsigma}_{m z}\right|,\left|\ddot{\varsigma}_{m z}\right|\right\} ; \quad \hat{\rho}_{m z}=$ $\max \left\{\left|\dot{\rho}_{m z}\right|,\left|\ddot{\rho}_{m z}\right|\right\} ; \quad \hat{\beta}_{m z}=\max \left\{\left|\dot{\beta}_{m z}\right|,\left|\ddot{\beta}_{m z}\right|\right\}$ and $\hat{\omega}_{m z}=\max \left\{\left|\dot{\omega}_{m z}\right|,\left|\ddot{\omega}_{m z}\right|\right\}$. According to Assumption 1, we can get $\left|f_{z}(a)\right| \leq M_{z}$ and $\left|g_{z}(a)\right| \leq N_{z}$ for any $a \in \Re$, where $M_{z}$ and $N_{z}$ are positive constants. Therefore, we can obtain

$$
\begin{aligned}
& \sum_{m=1}^{l} r^{2}(t) e_{m}(t)\left\{\sum_{z=1}^{l}\left[\varsigma_{m z}\left(x_{m}(t)\right)+\Delta \varsigma_{m z}(t)\right] f_{z}\left(x_{z}(t)\right)\right. \\
& \left.-\sum_{z=1}^{l}\left[\omega_{m z}\left(y_{m}(t)\right)+\Delta \omega_{m z}(t)\right] f_{z}\left(y_{z}(t)\right)\right\} \\
& \leq \sum_{m=1}^{l} r^{2}(t)\left|e_{m}(t)\right| \sum_{z=1}^{l}\left\{\left[\left|\varsigma_{m z}\left(x_{m}(t)\right)\right|+\left|\Delta \varsigma_{m z}(t)\right|\right]\right. \\
& \left.\times\left|f_{z}\left(x_{z}(t)\right)\right|+\left[\left|\omega_{m z}\left(y_{m}(t)\right)\right|+\left|\Delta \omega_{m z}(t)\right|\right]\left|f_{z}\left(y_{z}(t)\right)\right|\right\} \\
& \leq \sum_{m=1}^{l} r^{2}(t)\left|e_{m}(t)\right| \sum_{z=1}^{l}\left(\hat{\varsigma}_{m z}+\mu_{m z}+\hat{\omega}_{m z}+\chi_{m z}\right) M_{z},
\end{aligned}
$$


and

$$
\begin{aligned}
& \sum_{m=1}^{l} r^{2}(t) e_{m}(t)\left\{\sum_{z=1}^{l}\left[\rho_{m z}\left(x_{m}(t)\right)+\Delta \rho_{m z}(t)\right]\right. \\
& \times g_{z}\left(x_{z}\left(t-\tau_{z}(t)\right)\right)-\sum_{z=1}^{l}\left[\beta_{m z}\left(y_{m}(t)\right)\right. \\
& \left.\left.+\Delta \beta_{m z}(t)\right] g_{z}\left(y_{z}\left(t-\tau_{z}(t)\right)\right)\right\} \\
& \leq \sum_{m=1}^{l} r^{2}(t)\left|e_{m}(t)\right| \sum_{z=1}^{l}\left\{\left[\left|\rho_{m z}\left(x_{m}(t)\right)\right|\right.\right. \\
& \left.+\left|\Delta \rho_{m z}(t)\right|\right]\left|g_{z}\left(x_{z}\left(t-\tau_{z}(t)\right)\right)\right| \\
& \left.+\left[\left|\beta_{m z}\left(y_{m}(t)\right)\right|+\left|\Delta \beta_{m z}(t)\right|\right]\left|g_{z}\left(y_{z}\left(t-\tau_{z}(t)\right)\right)\right|\right\} \\
& \leq \sum_{m=1}^{l} r^{2}(t)\left|e_{m}(t)\right| \sum_{z=1}^{l}\left(\hat{\rho}_{m z}+v_{m z}+\hat{\beta}_{m z}+\Upsilon_{m z}\right) N_{z} .
\end{aligned}
$$

From adaptive controller (A), $r(t)$ is a continuously differentiable and monotone nondecreasing function. $r(s)$ is a positive constant, $s \in[-\tau, 0]$, and $\lim _{t \rightarrow+\infty} r(t)=+\infty$. Moreover, the function $r(t)$ satisfies $\frac{\dot{r}(t)}{r(t)} \leq c$ in Assumption 2 , where $c=\min _{1 \leq m \leq l}\left\{c_{m}\right\}$. Then,

$$
\sum_{m=1}^{l}\left(\dot{r}(t)-c_{m} r(t)\right) r(t) e_{m}^{2}(t) \leq 0
$$

Therefore, we have

$$
\begin{aligned}
\dot{V}_{1}(t) & \leq \sum_{m=1}^{l}\left(\dot{r}(t)-c_{m} r(t)\right) r(t) e_{m}^{2}(t) \\
& +\sum_{m=1}^{l} r^{2}(t)\left|e_{m}(t)\right| \\
& \times\left[\sum _ { z = 1 } ^ { l } \left[\left(\hat{\varsigma}_{m z}+\mu_{m z}+\hat{\omega}_{m z}+\chi_{m z}\right) M_{z}\right.\right. \\
& \left.\left.+\left(\hat{\rho}_{m z}+v_{m z}+\hat{\beta}_{m z}+\Upsilon_{m z}\right) N_{z}\right]-\Theta_{m}\right] \\
& \leq-\sum_{m=1}^{l} r^{2}(t)\left|e_{m}(t)\right|\left\{\Theta_{m}\right. \\
& -\sum_{z=1}^{l}\left[\left(\hat{\varsigma}_{m z}+\mu_{m z}+\hat{\omega}_{m z}+\chi_{m z}\right) M_{z}\right. \\
& \left.\left.+\left(\hat{\rho}_{m z}+v_{m z}+\hat{\beta}_{m z}+\Upsilon_{m z}\right) N_{z}\right]\right\} .
\end{aligned}
$$

The positive constant $\Theta_{m}$ can be properly chosen as

$$
\begin{aligned}
\Theta_{m} & =\sum_{z=1}^{l}\left[\left(\hat{\varsigma}_{m z}+\mu_{m z}+\hat{\omega}_{m z}+\chi_{m z}\right) M_{z}\right. \\
& \left.+\left(\hat{\rho}_{m z}+v_{m z}+\hat{\beta}_{m z}+\Upsilon_{m z}\right) N_{z}\right]+1 .
\end{aligned}
$$

Thus, we get

$$
\dot{V}_{1}(t) \leq-\sum_{m=1}^{l} r^{2}(t)\left|e_{m}(t)\right| \leq 0 .
$$

From the mathematical expression of $V_{1}(t)$, we have

$$
\frac{1}{2} r^{2}(t) \sum_{m=1}^{l} e_{m}^{2}(t) \leq V_{1}(t) \leq V_{1}(0)
$$

and

$$
\begin{aligned}
V_{1}(0) & =\frac{1}{2} \sum_{m=1}^{l} r^{2}(0) e_{m}^{2}(0)+\sum_{m=1}^{l} \frac{1}{2 \theta_{m}} \lambda_{m}^{2}(0) \\
+ & \sum_{m=1}^{l} \frac{1}{2 \sigma_{m}}\left(\varepsilon_{m}(0)-\Theta_{m}\right)^{2}
\end{aligned}
$$

If $\sup _{-\tau \leq s \leq 0}\|e(s)\|^{2} \neq 0$, there exists a positive constant $\eta$, such that

$\sum_{m=1}^{l} \frac{1}{2 \theta_{m}} \lambda_{m}^{2}(0)+\sum_{m=1}^{l} \frac{1}{2 \sigma_{m}}\left(\varepsilon_{m}(0)-\Theta_{m}\right)^{2} \leq \eta \sup _{-\tau \leq s \leq 0}\|e(s)\|^{2}$.

Thus, we can obtain

$$
\frac{1}{2} r^{2}(t)\|e(t)\|^{2} \leq\left[\frac{1}{2} r^{2}(0)+\eta\right] \sup _{-\tau \leq s \leq 0}\|e(s)\|^{2},
$$

and

$$
\|e(t)\| \leq P_{1} \sup _{-\tau \leq s \leq 0}\|e(s)\| / r(t),
$$

where $P_{1}=\left[r^{2}(0)+2 \eta\right]^{1 / 2}>0$. From Definition 4 , MNN systems (5) and (4) can achieve robust multimode function synchronization with adaptive controller (A). For convenience, we set $c=1$. When $r(t)$ is a polynomial function, such as $r(t)=t^{2}+1, \frac{\dot{r}(t)}{r(t)}=\frac{2 t}{t^{2}+1} \leq c$. Then system (5) and system (4) can achieve robust polynomial synchronization with the adaptive controller (A). When $r(t)$ is a logarithmic function, such as $r(t)=\ln \left(t^{2}+10\right), \frac{\dot{r}(t)}{r(t)}=\frac{2 t}{\left(t^{2}+10\right) \ln \left(t^{2}+10\right)} \leq c$. Then system (5) and system (4) can achieve robust logarithmical synchronization with the adaptive controller (A). The proof is finished.

\section{B. Proof of Corollary 1}

Proof. Consider a Lyapunov function:

$$
\begin{aligned}
V_{2}(t) & =\frac{1}{2} \sum_{m=1}^{l} e^{2 b t} e_{m}^{2}(t)+\sum_{m=1}^{l} \frac{1}{2 \theta_{m}}\left(\lambda_{m}(t)-\delta_{m}\right)^{2} \\
& +\sum_{m=1}^{l} \frac{1}{2 \sigma_{m}}\left(\varepsilon_{m}(t)-\Theta_{m}\right)^{2}
\end{aligned}
$$

where $\delta_{m}$ and $\Theta_{m}$ are positive constants that will be decided.

Combining the derivative of $V_{2}(t)$ with the proof of Theorem 1, we get:

$$
\begin{aligned}
\dot{V}_{2}(t) & \leq e^{2 b t} \sum_{m=1}^{l}\left(b-c_{m}-\delta_{m}\right) e_{m}^{2}(t)+e^{2 b t} \sum_{m=1}^{l}\left|e_{m}(t)\right| \\
& \times\left[\sum _ { z = 1 } ^ { l } \left[\left(\hat{\varsigma}_{m z}+\mu_{m z}+\hat{\omega}_{m z}+\chi_{m z}\right) M_{z}\right.\right. \\
& \left.\left.+\left(\hat{\rho}_{m z}+v_{m z}+\hat{\beta}_{m z}+\Upsilon_{m z}\right) N_{z}\right]-\Theta_{m}\right]
\end{aligned}
$$

The positive constants $\delta_{m}$ and $\Theta_{m}$ can be properly chosen as

$$
\delta_{m}=b-c_{m}
$$

and

$$
\begin{aligned}
\Theta_{m} & =\sum_{z=1}^{l}\left[\left(\hat{\varsigma}_{m z}+\mu_{m z}+\hat{\omega}_{m z}+\chi_{m z}\right) M_{z}\right. \\
& \left.+\left(\hat{\rho}_{m z}+v_{m z}+\hat{\beta}_{m z}+\Upsilon_{m z}\right) N_{z}\right]+1 .
\end{aligned}
$$

Then we can obtain

$$
\dot{V}_{2}(t) \leq-e^{2 b t} \sum_{m=1}^{l}\left|e_{m}(t)\right| \leq 0 .
$$

Therefore, when $r(t)$ is an exponential function, MNN systems (5) and (4) can realize robust exponential synchronization under adaptive controller (A) with no need for the assumption 2. 


\section{Proof of Theorem 2}

Proof: Consider a Lyapunov function:

$$
\begin{aligned}
& V_{3}(t)=\frac{1}{2} \sum_{m=1}^{l}\left[r(t) e_{m}(t)\right]^{2} \\
& +\sum_{m=1}^{l} \int_{t-\tau_{m}(t)}^{t}\left[r(s) h_{m}\left(e_{m}(s)\right)\right]^{2} d s \\
& +\sum_{m=1}^{l} \frac{1}{2 \theta_{m}}\left(\lambda_{m}(t)-\delta_{m}\right)^{2}+\sum_{m=1}^{l} \frac{1}{2 \sigma_{m}}\left(\varepsilon_{m}(t)-\Theta_{m}\right)^{2},
\end{aligned}
$$

where $\delta_{m}$ and $\Theta_{m}$ are positive constants that will be decided.

The derivative of $V_{3}(t)$ can be written as:

$$
\begin{aligned}
& \dot{V}_{3}(t)=\sum_{m=1}^{l} r(t) \dot{r}(t) e_{m}^{2}(t)+\sum_{m=1}^{l} r^{2}(t) e_{m}(t) \\
& \times\left\{-c_{m} e_{m}(t)+\sum_{z=1}^{l}\left[\varsigma_{m z}\left(x_{m}(t)\right)+\Delta \varsigma_{m z}(t)\right] f_{z}\left(x_{z}(t)\right)\right. \\
& -\sum_{z=1}^{l}\left[\omega_{m z}\left(y_{m}(t)\right)+\Delta \omega_{m z}(t)\right] f_{z}\left(y_{z}(t)\right) \\
& +\sum_{z=1}^{l}\left[\rho_{m z}\left(x_{m}(t)\right)+\Delta \rho_{m z}(t)\right] g_{z}\left(x_{z}\left(t-\tau_{z}(t)\right)\right) \\
& -\sum_{z=1}^{l}\left[\beta_{m z}\left(y_{m}(t)\right)+\Delta \beta_{m z}(t)\right] g_{z}\left(y_{z}\left(t-\tau_{z}(t)\right)\right) \\
& \left.-\lambda_{m}(t) h_{m}\left(e_{m}(t)\right)-\varepsilon_{m}(t) \operatorname{sgn}\left(e_{m}(t)\right)\right\} \\
& +\sum_{m=1}^{l}\left[r(t) h_{m}\left(e_{m}(t)\right)\right]^{2} \\
& -\sum_{m=1}^{l}\left[r\left(t-\tau_{m}(t)\right) h_{m}\left(e_{m}\left(t-\tau_{m}(t)\right)\right)\right]^{2}\left(1-\dot{\tau}_{m}(t)\right) \\
& +\sum_{m=1}^{l}\left(\lambda_{m}(t)-\delta_{m}\right) r^{2}(t) e_{m}(t) h_{m}\left(e_{m}(t)\right) \\
& +\sum_{m=1}^{l}\left(\varepsilon_{m}(t)-\Theta_{m}\right) r^{2}(t)\left|e_{m}(t)\right| \\
& =\sum_{m=1}^{l} r(t) \dot{r}(t) e_{m}^{2}(t)+\sum_{m=1}^{l} r^{2}(t) e_{m}(t) \\
& \times\left\{\sum_{m} e_{m}(t)+\sum_{z=1}^{l}\left[\varsigma_{m z}\left(x_{m}(t)\right)+\Delta \varsigma_{m z}(t)\right] f_{z}\left(x_{z}(t)\right)\right. \\
& +\sum_{z=1}^{l}\left[\omega_{m z}\left(y_{m}(t)\right)+\Delta \omega_{m z}(t)\right] f_{z}\left(y_{z}(t)\right) \\
& +\sum_{z=1}^{l}\left[\rho_{m z}\left(x_{m}(t)\right)+\Delta \rho_{m z}(t)\right] g_{z}\left(x_{z}\left(t-\tau_{z}(t)\right)\right) \\
& \left.-\sum_{z=1}^{l}\left[\beta_{m z}\left(y_{m}(t)\right)+\Delta \beta_{m z}(t)\right] g_{z}\left(y_{z}\left(t-\tau_{z}(t)\right)\right)\right\} \\
& +\sum_{m=1}^{l}\left[r(t) h_{m}\left(e_{m}(t)\right)\right]^{2} \\
& -\sum_{m=1}^{l}\left[r\left(t-\tau_{m}(t)\right) h_{m}\left(e_{m}\left(t-\tau_{m}(t)\right)\right)\right]^{2}\left(1-\dot{\tau}_{m}(t)\right) \\
& -\sum_{m=1}^{l} \delta_{m} r^{2}(t) e_{m}(t) h_{m}\left(e_{m}(t)\right)-\sum_{m=1}^{l} \Theta_{m} r^{2}(t)\left|e_{m}(t)\right| \\
& \\
& +0 m A s s m p \\
&
\end{aligned}
$$

From Assumption 4, we get

$$
-e_{m}(t) h_{m}\left(e_{m}(t)\right) \leq \frac{-h_{m}^{2}\left(e_{m}(t)\right)}{L_{m}} .
$$

From Assumption 3, the transmission delay $\tau_{m}(t), m=$ $1,2, \ldots, l$, satisfies $\dot{\tau}_{m}(t) \leq 1$. Thus, we can obtain

$$
-\sum_{m=1}^{l}\left[r\left(t-\tau_{m}(t)\right) h_{m}\left(e_{m}\left(t-\tau_{m}(t)\right)\right)\right]^{2}\left(1-\dot{\tau}_{m}(t)\right) \leq 0 .
$$

From adaptive controller (B), $r(t)$ is a continuously differentiable and monotone nondecreasing function. $r(s)$ is a positive constant, $s \in[-\tau, 0]$, and $\lim _{t \rightarrow+\infty} r(t)=+\infty$. Moreover, the function $r(t)$ satisfies $\frac{\dot{r}(t)}{r(t)} \leq c$ in Assumption 2, where $c=\min _{1 \leq m \leq l}\left\{c_{m}\right\}$. Combining with the proof of Theorem 1 , we have

$$
\begin{aligned}
& \dot{V}_{3}(t) \leq \sum_{m=1}^{l}\left[\dot{r}(t)-c_{m} r(t)\right] r(t) e_{m}^{2}(t)+\sum_{m=1}^{l} r^{2}(t)\left|e_{m}(t)\right| \\
& \times \sum_{z=1}^{l}\left[\left(\hat{\varsigma}_{m z}+\mu_{m z}+\hat{\omega}_{m z}+\chi_{m z}\right) M_{z}\right. \\
& \left.+\left(\hat{\rho}_{m z}+v_{m z}+\hat{\beta}_{m z}+\Upsilon_{m z}\right) N_{z}\right] \\
& +\sum_{m=1}^{l}\left[r(t) h_{m}\left(e_{m}(t)\right)\right]^{2}-\sum_{m=1}^{l} \delta_{m} r^{2}(t) e_{m}(t) h_{m}\left(e_{m}(t)\right) \\
& -\sum_{m=1}^{l} \Theta_{m} r^{2}(t)\left|e_{m}(t)\right| \\
& \leq \sum_{m=1}^{l}\left(1-\frac{\delta_{m}}{L_{m}}\right)\left[r(t) h_{m}\left(e_{m}(t)\right)\right]^{2}+\sum_{m=1}^{l} r^{2}(t)\left|e_{m}(t)\right| \\
& \times\left\{\sum _ { z = 1 } ^ { l } \left[\left(\hat{\varsigma}_{m z}+\mu_{m z}+\hat{\omega}_{m z}+\chi_{m z}\right) M_{z}\right.\right. \\
& \left.\left.+\left(\hat{\rho}_{m z}+v_{m z}+\hat{\beta}_{m z}+\Upsilon_{m z}\right) N_{z}\right]-\Theta_{m}\right\}
\end{aligned}
$$

The positive constants $\delta_{m}$ and $\Theta_{m}$ can be properly chosen as

$$
\delta_{m}=L_{m},
$$

and

$$
\begin{aligned}
\Theta_{m} & =\sum_{z=1}^{l}\left[\left(\hat{\varsigma}_{m z}+\mu_{m z}+\hat{\omega}_{m z}+\chi_{m z}\right) M_{z}\right. \\
& \left.+\left(\hat{\rho}_{m z}+v_{m z}+\hat{\beta}_{m z}+\Upsilon_{m z}\right) N_{z}\right]+1 .
\end{aligned}
$$

Thus, we can obtain

$$
\dot{V}_{3}(t) \leq-\sum_{m=1}^{l} r^{2}(t)\left|e_{m}(t)\right| \leq 0 .
$$

From (23), we can have

$$
\frac{1}{2} r^{2}(t) \sum_{m=1}^{l} e_{m}^{2}(t) \leq V_{3}(t) \leq V_{3}(0)
$$

and

$$
\begin{aligned}
& V_{3}(0)=\frac{1}{2} \sum_{m=1}^{l} r^{2}(0) e_{m}^{2}(0)+\sum_{m=1}^{l} \int_{-\tau_{m}(0)}^{0} r^{2}(s) h_{m}^{2}\left(e_{m}(s)\right) d s \\
& +\sum_{m=1}^{l} \frac{1}{2 \theta_{m}}\left(\lambda_{m}(0)-\delta_{m}\right)^{2}+\sum_{m=1}^{l} \frac{1}{2 \sigma_{m}}\left(\varepsilon_{m}(0)-\Theta_{m}\right)^{2} .
\end{aligned}
$$

If $\sup _{-\tau \leq s \leq 0}\|e(s)\|^{2} \neq 0$, there exists a positive constant $\pi$, such that

$$
\begin{aligned}
& \sum_{m=1}^{l} \frac{1}{2 \theta_{m}}\left(\lambda_{m}(0)-\delta_{m}\right)^{2}+\sum_{m=1}^{l} \frac{1}{2 \sigma_{m}}\left(\varepsilon_{m}(0)-\Theta_{m}\right)^{2} \\
& \leq \pi \\
& \sup _{-\tau \leq s \leq 0}\|e(s)\|^{2} .
\end{aligned}
$$


Then

$$
\begin{aligned}
& V_{3}(0) \leq \frac{1}{2} r^{2}(0)\|e(0)\|^{2} \\
& +\max _{1 \leq m \leq l}\left\{L_{m}^{2}\right\} \sum_{m=1}^{l} \int_{-\tau_{m}(0)}^{0} r^{2}(s) e_{m}^{2}(s) d s \\
& +\pi \sup _{-\tau \leq s \leq 0}\|e(s)\|^{2} \\
& \leq\left[\frac{1}{2} r^{2}(0)+\tau \max _{-\tau \leq s \leq 0 ; 1 \leq m \leq l}\left\{r^{2}(s) L_{m}^{2}\right\}+\pi\right] \\
& \times \sup _{-\tau \leq s \leq 0}\|e(s)\|^{2} .
\end{aligned}
$$

Thus, we can have

$$
\begin{aligned}
& \frac{1}{2} r^{2}(t)\|e(t)\|^{2} \\
& \leq\left[\frac{1}{2} r^{2}(0)+\tau \max _{-\tau \leq s \leq 0 ; 1 \leq m \leq l}\left\{r^{2}(s) L_{m}^{2}\right\}+\pi\right] \\
& \times \sup _{-\tau \leq s \leq 0}\|e(s)\|^{2}
\end{aligned}
$$

and

$$
\|e(t)\| \leq P_{2} \sup _{-\tau \leq s \leq 0}\|e(s)\| / r(t)
$$

where $P_{2}=\left[r^{2}(0)+2 \tau \max _{-\tau \leq s \leq 0 ; 1 \leq m \leq l}\left\{r^{2}(s) L_{m}^{2}\right\}+2 \pi\right]^{\frac{1}{2}}$ $>0$. From Definition 4, MNN systems (5) and (4) can achieve multimode function synchronization with adaptive controller (B). More specifically, when $r(t)$ is an exponential function, such as $r(t)=e^{b t}, 0<b \leq c$, MNN systems (5) and (4) can realize robust exponential synchronization under adaptive controller (B). For convenience, we set $c=1$. When $r(t)$ is a polynomial function, such as $r(t)=t^{2}+1$, system (5) and system (4) can achieve robust polynomial synchronization with adaptive controller (B). When $r(t)$ is a logarithmic function, such as $r(t)=\ln \left(t^{2}+10\right)$, system (5) and system (4) can achieve robust logarithmical synchronization with adaptive controller (B). The proof is finished.

\section{REFERENCES}

[1] L. Chua, "Memristor-the missing circuit element," IEEE Trans.Circuit Theory, vol. 18, no. 5, pp. 507-519, 1971.

[2] D. Strukov, G. Snider, D. Stewart, and R. Williams, "The missing memristor found," Nature, vol. 453, pp. 80-83, 2008.

[3] L. Zhou, C. Wang, and L. Zhou, "A novel no-equilibrium hyperchaotic multi-wing system via introducing memristor," Int. J. Circ. Theor. Appl, vol.46, no. 1, pp. 84-98, 2018

[4] Q. Zhao, C. Wang, and X. Zhang, "A universal emulator for memristor, memcapacitor, and meminductor and its chaotic circuit," Chaos, vol. 29, no. 1, pp. 013141-1-013141-14, 2019.

[5] C. Wang, X. Liu and H. Xia, "Multi-piecewise quadratic nonlinearity memristor and its $2 \mathrm{~N}$-scroll and $2 \mathrm{~N}+1$-scroll chaotic attractors system," Chaos, vol. 27, no. 3, pp. 033114-1-033114-12, 2017.

[6] S. Wen, R. Hu, Y. Yang, et al. "Memristor-based echo state network with online least mean square," IEEE Trans. Syst., Man, Cybern., Syst., vol. 49, no. 9, pp. 1787-1796, 2019.

[7] A. Buscarino, C. Corradino, L. Fortuna, and L. Chua, "Taming spatiotemporal chaos in forced memristive arrays," IEEE Trans. VLSI Syst., pp. 1-8, 2018.

[8] L. Zhou, C. Wang, and L. Zhou, "Generating four-wing hyperchaotic attractor and two-wing, three-wing, and four-wing chaotic attractors in $4 \mathrm{D}$ memristive system," Int. J. Bifurcation Chaos, vol. 27, no. 2, pp.17500271-1750027-14, 2017.

[9] C. Wang, L. Xiong, J. Sun, and W. Yao, "Memristor-based neural networks with weight simultaneous perturbation training," Nonlinear Dyn., to be published, doi: 10.1007/s11071-018-4730-z.

[10] R. Wu, and C. Wang, "A new simple chaotic circuit based on memristor," Int. J. Bifurcation Chaos, vol. 26, no. 9, pp. 1650145-1-1650145-11, 2016.
[11] L. Zhou, C. Wang, X. Zhang, and W. Yao, "Various attractors, coexisting attractors and antimonotonicity in a simple fourth-order memristive twinT oscillator," Int. J. Bifurcation Chaos, vol. 28, no. 4, pp. 1850050-11850050-18, 2018.

[12] T. Li, S. Duan, J. Liu, et al. "A spintronic memristor-based neural network with radial basis function for robotic manipulator control implementation," IEEE Trans. Syst., Man, Cybern., Syst., vol. 46, no. 4, pp. 582-588, 2016

[13] M. Itoh and L. O. Chua, "Memristor cellular automata and memristor discrete-time cellular neural networks," Int. J. Bifurcat. Chaos, vol. 19, no. 11, pp. 3605-3656, 2009.

[14] S. Duan, X. Hu, Z. Dong, L. Wang, and P. Mazumder, "Memristor-based cellular nonlinear/neural network: design, analysis, and applications," IEEE Trans. Neural Netw. Learn. Syst., vol. 26, no. 6, pp. 1202-1213, Jun. 2015.

[15] M. Rubenstein, A. Cornejo, and R. Nagpal, "Programmable selfassembly in a thousand-robot swarm," Science, vol. 345, pp. 795-799, 2014.

[16] L. Shanmugam, P. Mani, R. Rajan, and Y. Joo, "Adaptive synchronization of reaction-diffusion neural networks and its application to secure communication," IEEE Trans. Cybern., to be published, doi: 10.1109/TCYB.2018.2877410.

[17] Y. Xia, Z. Yang, and M. Han, "Lag synchronization of unknown chaotic delayed Yang-Yang-type fuzzy neural networks with noise perturbation based on adaptive control and parameter identification," IEEE Trans. Neural Netw., vol. 20, no. 7, pp. 1165-1180, Jun. 2009.

[18] S. Wen, Z. Zeng, T. Huang, and Y. Zhang, "Exponential adaptive lag synchronization of memristive neural networks via fuzzy method and applications in pseudorandom number generators," IEEE Trans. Fuzzy Syst., vol. 22, no. 6, pp. 1704-1713, Dec. 2014

[19] N. Li, and J. Cao, "Lag synchronization of memristor-based coupled neural networks via $\omega$-measure," IEEE Trans. Neural Netw. Learn. Syst., vol. 27, no. 3, pp. 686-697, Mar. 2016.

[20] L. Li and J. Cao, "Cluster synchronization in an array of coupled stochastic delayed neural networks via pinning control," Neurocomputing, vol. 74, no. 5, pp. 846-856, 2011.

[21] Y. W. Wang, W. Yang, J. W. Xiao, and Z. G. Zeng, "Impulsive multisynchronization of coupled multistable neural networks with timevarying delay," IEEE Trans. Neural Netw. Learn. Syst., vol. 28, no. 7, pp. 1560-1571, Jul. 2017.

[22] J. E Zhang, "Multisynchronization for coupled multistable fractionalorder neural networks via impulsive control," Complexity, vol. 2017, no. 9323172, pp. 1-10, 2017.

[23] X. Lv, X. Li, J. Cao, et al. "Dynamical and static multisynchronization of coupled multistable neural networks via impulsive control," IEEE Trans. Neural Netw. Learn. Syst., vol. 29, no. 12, pp. 6062-6072, 2018.

[24] H. Wu, R. Li, R. Yao, and X. Zhang, "Weak, modified and function projective synchronization of chaotic memristive neural networks with time delays," Neurocomputing, vol. 149, pp. 667-676, 2015.

[25] Y. Fan, X. Huang, Y. Li, et al. "Aperiodically intermittent control for quasi-synchronization of delayed memristive neural networks: an interval matrix and matrix measure combined method," IEEE Trans. Syst., Man, Cybern., Syst., vol. 49, no. 11, pp. 2254-2265, 2019.

[26] A. Wu, S. Wen, and Z. Zeng, "Synchronization control of a class of memristor-based recurrent neural networks," Inf. Sci., vol. 183, no. 1, pp. 106-116, 2012

[27] S. Wen, G. Bao, Z. Zeng, Y. Chen and T. Huang, "Global exponential synchronization of memristor-based recurrent neural networks with timevarying delays," Neural Netw., vol. 48, pp. 195-203, Dec. 2013.

[28] W. Guan, S. Yi, and Y. Quan, "Exponential synchronization of coupled memristive neural networks via pinning control," Chin. Phys. B, vol. 22, no. 5, 2013.

[29] G. Wang and Y. Shen, "Exponential synchronization of coupled memristive neural networks with time delays," Neural Comput. Appl., vol. 24, no. 6, pp. 1421-1430, 2014.

[30] A. Wu, Z. Zeng, X. Zhu, and J. Zhang, "Exponential synchronization of memristor-based recurrent neural networks with time delays," Neurocomputing, vol. 74, no. 17, pp. 3043-3050, 2011.

[31] Z. Guo, J. Wang, and Z. Yan, "Global exponential synchronization of two memristor-based recurrent neural networks with time delays via static or dynamic coupling," IEEE Trans. Syst., Man, Cybern., Syst., vol. 45, no. 2, pp. 235-249, Feb. 2015.

[32] L. Wang, Y. Shen, Q. Yin, and G. Zhang, "Adaptive synchronization of memristor-based neural networks with time-varying delays," IEEE Trans. Neural Netw. Learn. Syst., vol. 26, no. 9, pp. 2033-2042, Sep. 2015. 
[33] G. Zhang, Y. Shen, and L. Wang, "Global anti-synchronization of a class of chaotic memristive neural networks with time-varying delays," Neural Netw., vol. 36, pp. 1-8, Nov. 2013.

[34] A. Wu and Z. Zeng, "Anti-synchronization control of a class of memristive recurrent neural networks," Commun. Nonlin. Sci. Numer. Simul., vol. 18, no. 2, pp. 373-385, Feb. 2013.

[35] W. Zhang, C. Li, T. Huang, and X. He, "Synchronization of memristorbased coupling recurrent neural networks with time-varying delays and impulses," IEEE Trans. Neural Netw. Learn. Syst., vol. 26, no. 12, pp. 3308-3313, Dec. 2015.

[36] M. Zheng, L. Li, H. Peng, et al. "Finite-time projective synchronization of memristor-based delay fractional-order neural networks," Nonlinear Dyn., vol. 89, no. 4, pp. 2641-2655, 2017

[37] X. Yang, J. Cao and J. Liang, "Exponential synchronization of memristive neural networks with delays: interval matrix method," IEEE Trans. Neural Netw. Learn. Syst., vol. 28, no. 8, pp. 1878-1888, 2017.

[38] Y. Li, B. Luo, D. Liu, and Z. Yang, "Robust synchronization of memristive neural networks with strong mismatch characteristics via pinning control," Neurocomputing, vol. 289, pp. 144-154, 2018.

[39] X. Yang and D. Ho, "Synchronization of delayed memristive neural networks: robust analysis approach," IEEE Trans. Cybern., vol. 46, no. 12, pp. 3377-3387, 2016.

[40] H. Zhao, L. Li, H. Peng, et al. "Finite-time robust synchronization of memristive neural network with perturbation," Neural Process Lett., vol. 47, pp. 509-533, 2018.

[41] S. Yang, Z. Guo, and J. Wang, "Robust synchronization of multiple memristive neural networks with uncertain parameters via nonlinear coupling," IEEE Trans. Syst., Man, Cybern., Syst., vol. 45, no. 7, pp. 1077-1086, 2015

[42] K. Guan, F. Tan, and J. Yang, "Global power synchronization of complex dynamical networks with proportional delay and impulsive effects," Neurocomputing, vol. 366, pp. 23-34, 2019.

[43] C. Kinh, L. Hien, and T. Ke, "Power-rate synchronization of fractionalorder nonautonomous neural networks with heterogeneous proportional delays," Neural Process Lett., vol. 47, no. 1, pp. 139-151, 2018.

[44] S. Gong, S. Yang, Z. Guo, and T. Huang, "Global exponential synchronization of memristive competitive neural networks with time-varying delay via nonlinear control," Neural Process Lett., vol. 49, no. 1, pp. 103-119, 2019.

[45] R. Li, X. Gao, and J. Cao, "Exponential synchronization of stochastic memristive neural networks with time-varying delays," Neural Process Lett., vol. 50, no. 1, pp. 459-475, 2019.

[46] H. Cheng, S. Zhong, X. Li, and Q. Zhong, "Exponential synchronization of delayed memristor-based neural networks with stochastic perturbation via nonlinear control," Neurocomputing, vol. 340, pp. 90-98, 2019.

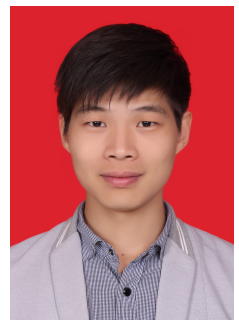

Wei Yao received the M.S. degree from College of Information Science and Engineering, Hunan University, Changsha, China, in 2014. He is currently pursuing the $\mathrm{Ph} . \mathrm{D}$ degree in Hunan University. His research interests mainly focus on nonlinear dynamical systems, complex systems and complex networks.

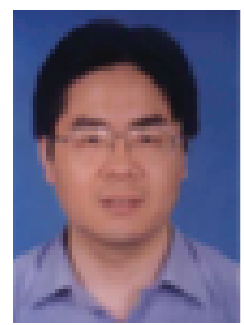

Chunhua Wang received the M.S. degree from Zhengzhou University, Zhengzhou, China, in 1994, and the Ph.D. degree from Beijing University of Technology, Beijing, China, in 2003.

$\mathrm{He}$ is currently the Professor of College of Information Science and Engineering, Hunan University, Changsha, China. He is the Doctor tutor, director of advanced communication technology key laboratory of hunan universities, the member of academic committee of hunan university, the director of chaos and nonlinear circuit professional committee of circuit and system branch of China electronic society. Now, his research interests include memristor circuit, complex networks, chaotic circuit, chaos secure communication, current-mode circuit and neural networks based on memristor. $\mathrm{He}$ has presided over 8 national and provincial projects, and published more than 120 papers, among which more than 100 were retrieved by SCI.

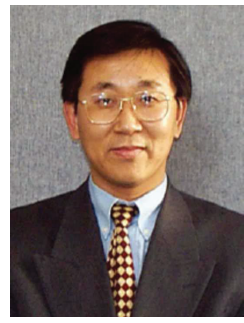

Yichuang Sun (M'90-SM'99) received the B.Sc. and M.Sc. degrees from Dalian Maritime University, Dalian, China, in 1982 and 1985, respectively, and the Ph.D. degree from the University of York, York, U.K., in 1996, all in communications and electronics engineering.

He is currently a Professor and the HoD of Electronic, Communication and Electrical Engineering with the School of Engineering and Technology, University of Hertfordshire, U.K. He has published over 320 papers and contributed 10 chapters in edited books. He has also published four text and research books: Continuoustime Active Filter Design (CRC Press, USA, 1999), Design of High frequency Integrated Analogue Filters (IEE Press, U.K., 2002), Wireless Communication Circuits and Systems (IET Press, 2004), and Test and Diagnosis of Analogue, Mixed-signal and RF Integrated Circuits-the Systems on Chip Approach (IET Press, 2008). His research interests are mainly in the areas of wireless and mobile communications, microelectronic circuits and systems, and machine learning for communications and electronics.

$\mathrm{He}$ was a Series Editor of the IEE Circuits, Devices and Systems Book Series from 2003 to 2008. He has been Associate Editor of the IEEE TRANSACTIONS ON CIRCUITS AND SYSTEMS I: REGULAR PAPERS from 2010 to 2011, from 2016 to 2017, and from 2018 to 2019. He is also an Editor of the ETRI Journal, Journal of Semiconductors, and some others. He was a Guest Editor of eight IEEE and IEE/IET journal special issues: High frequency Integrated Analogue Filters in IEE Proc. Circuits, Devices and Systems (2000), RF Circuits and Systems for Wireless Communications in IEE Proc. Circuits, Devices and Systems (2002), Analogue and Mixed Signal Test for Systems on Chip in IEE Proc. Circuits, Devices and Systems (2004), MIMO Wireless and Mobile Communications in IEE Proc. Communications (2006), Advanced Signal Processing for Wireless and Mobile Communications in IET Signal Processing (2009), Cooperative Wireless and Mobile Communications in IET Communications (2013), Software-Defined Radio Transceivers and Circuits for 5G Wireless Communications in IEEE Transactions on Circuits and Systems-II (2016), and 2016 IEEE International Symposium on Circuits and Systems in IEEE Transactions on Circuits and Systems-I (2016). He has also been widely involved in various IEEE technical committee and international conference activities.

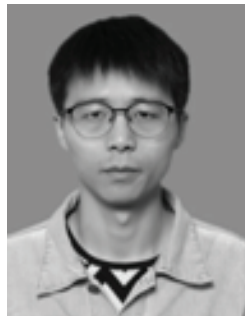

Chao Zhou received the B.S. degree in automation from the $\mathrm{Xi}^{\prime}$ an University of Science and Technology, Xi'an, China, in 2014, and the M.S. degree in control science and engineering from Hunan University, Changsha, China, in 2017, where he is currently pursuing the $\mathrm{Ph} . \mathrm{D}$. degree in computer science and technology. His current research interests include the nonlinear dynamical systems, complex systems and complex networks. 